\title{
Coriolis coupling as a source of non-RRKM effects in triatomic near-symmetric top molecules: Diffusive intramolecular energy exchange between rotational and vibrational degrees of freedom
}

\author{
M. Kryvohuz and R. A. Marcus ${ }^{\text {a) }}$ \\ Noyes Laboratories, California Institute of Technology, 127-72, Pasadena, California 91125, USA
}

(Received 27 October 2009; accepted 7 April 2010; published online 8 June 2010)

\begin{abstract}
A classical theory is proposed to describe the non-RRKM effects in activated asymmetric top triatomic molecules observed numerically in classical molecular dynamics simulations of ozone. The Coriolis coupling is shown to result in an effective diffusive energy exchange between the rotational and vibrational degrees of freedom. A stochastic differential equation is obtained for the $K$-component of the rotational angular momentum that governs the diffusion. (C) 2010 American Institute of Physics. [doi:10.1063/1.3430508]
\end{abstract}

\section{INTRODUCTION}

It was shown ${ }^{1-3}$ that the unusual mass-independent isotope effect in ozone formation ${ }^{4-6}$ can be explained by assuming some non-RRKM (non-Rice-Ramsperger-KasselMarcus) behavior of energetic ozone molecules. The reader can find a review of literature on the ozone isotope effect and the current status of theoretical investigations in Ref. 7. The non-RRKM behavior of vibrationally excited ozone molecules was suggested in several classical mechanical numerical studies and an important role of the coupling of the rotational degrees of freedom to the vibrations was emphasized. ${ }^{7,8}$ Recently, the classical molecular dynamics (MD) simulations ${ }^{9,10}$ of collisional ozone formation $\mathrm{O}+\mathrm{O}_{2}$ $\rightarrow \mathrm{O}_{3}^{*}$ showed a nonstatistical distribution of lifetimes of the vibrationally excited ozone molecules. In a series of two papers we show analytically that the observed non-RRKM effects are indeed governed by the Coriolis coupling between the rotational and vibrational degrees of freedom, which cannot be accounted for by the standard RRKM theory. ${ }^{11}$

The RRKM theory assumes that any degree of freedom can be treated as active or adiabatic. ${ }^{12}$ The former participates in the fast energy redistribution between the other degrees of freedom, while the latter remains in the same quantum state during the reaction and does not randomly exchange its energy with active states. ${ }^{13}$ The RRKM theory cannot describe the situation when the exchange of energy during the time of reaction is neither fast nor slow. An example of such process can be energy diffusion, when energy slowly diffuses from one degree of freedom to another. The reason for such slow diffusion can be in a moderate coupling between the corresponding states.

Rotational degrees of freedom are usually considered to be coupled either strongly or weakly to vibrational degrees of freedom and thus rotational degrees of freedom are considered as either active or adiabatic. Yet, in ozone molecule, the Coriolis coupling turns out to be strong enough to violate the assumption of adiabaticity and weak enough to consider

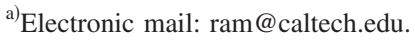

rotational degrees of freedom as active. ${ }^{8}$ The slow energy exchange between the vibrational and rotational degrees of freedom in ozone molecule was observed in the classical numerical simulations in Ref. 8. There it was indicated that the basic assumptions of the RRKM theory of the microcanonical energy redistribution between the vibrational and rotational degrees of freedom fail for ozone.

In the present paper we apply a classical analysis and show that the energy exchange between the rotational and vibrational degrees of freedom in an activated triatomic molecule such as ozone can be described by the process of energy diffusion. We start with exact equations for the softbody rotational dynamics and derive an effective stochastic differential equation that analytically describes the process of energy diffusion. The diffusion is shown to be fast enough to cause significant non-RRKM effects observed in Refs. 7-10. The derived theory is general for the wide class of triatomic molecules rotational dynamics of which can be described within the symmetric top approximation.

This paper is organized as follows. In Sec. II we review the standard approach to the treatment of molecular rotations as rigid body rotations. In Sec. III we derive equations for the soft-body rotational dynamics. In Secs. IV-VI we simplify the dynamics of soft-body rotations to the process of one-dimensional diffusion. We derive corrections to the symmetric top approximation in Sec. VII. In Sec. VIII we discuss the main effects of the observed diffusion. We indicate the effect of molecular symmetry on the Coriolis coupling in Sec. IX. The numerical algorithms used in the paper are described in Sec. X. In Sec. XI we conclude summarizing the main results of the work.

\section{RIGID BODY DYNAMICS}

In this section we review the general approach to the treatment of rotations of a triatomic molecule representing it as a rigid rotor. The equations of motion of a rigid body have the simplest form in its principal axes of inertia. ${ }^{14}$ For a nonlinear triatomic molecule such as ozone, axes $\xi_{1}$ and $\xi_{2}$ lie in the plane of the molecule, while axis $\xi_{3}$ is orthogonal to 


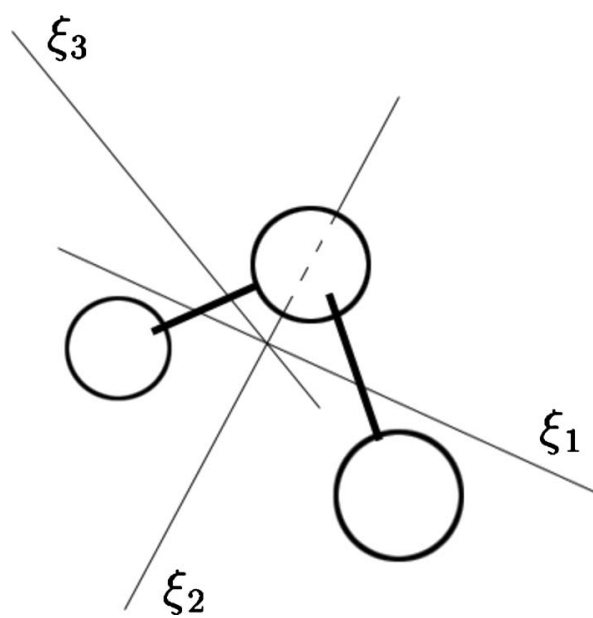

FIG. 1. Principal axes of inertia for a nonlinear three-atomic molecule.

the plane, Fig. 1 . We denote by $J_{1}, J_{2}$, and $J_{3}$ the projections of angular momentum $\mathbf{J}$ to these axes and introduce the principal moments of inertia $I_{1}, I_{2}$, and $I_{3}$ which correspond to these axes, respectively. Hereafter in the present paper the numeration of principal axes and corresponding principal values are chosen in such a way that $I_{1}<I_{2}<I_{3}$. The rotational energy of a molecule then reads

$$
E_{\mathrm{rot}}=\frac{J_{1}^{2}}{2 I_{1}}+\frac{J_{2}^{2}}{2 I_{2}}+\frac{J_{3}^{2}}{2 I_{3}} \text {. }
$$

The equilibrium geometry of the molecule has a valence bond angle which, in the case of ozone, is equal to $120^{\circ}$. This gives the ratio of the principal inertia moments $I_{1}: I_{2}: I_{3}$ $=1: 9: 10$. Since $I_{1} \ll I_{2}$ and for the planar molecule $I_{3}=I_{1}$ $+I_{2} \approx I_{2}$, then such a molecule is often considered as a symmetric top with $I_{2}^{\prime}=I_{3}^{\prime}=\sqrt{I_{2} I_{3}}$. This approximation converts expression (1) into

$$
E_{\mathrm{rot}}=\frac{J^{2}}{2 I_{2}^{\prime}}+\left(\frac{1}{2 I_{1}}-\frac{1}{2 I_{2}^{\prime}}\right) K^{2},
$$

where we used $J^{2}=J_{1}^{2}+J_{2}^{2}+J_{3}^{2}$ and defined $K \equiv J_{1}$. The minimum rotational energy for a given value of $J$ corresponds to $E_{\mathrm{rot}}^{\min }=J^{2} / 2 I_{2}^{\prime}$ and the maximum rotational energy corresponds to $E_{\mathrm{rot}}^{\max }=\left[\left(1 / 2 I_{1}\right)-\left(1 / 2 I_{2}^{\prime}\right)\right] J^{2}$, which in the case of ozone molecule results in $E_{\mathrm{rot}}^{\max }=\left[\left(I_{2}^{\prime}-I_{1}\right) / I_{1}\right] E_{\mathrm{rot}}^{\min } \approx 10 E_{\mathrm{rot}}^{\min }$. The value of $K$ for such molecules therefore significantly controls the amount of rotational energy for a given $J$. This simple observation plays an important role in the theory of non-RRKM effects developed in sections III-IX.

From the Euler equations ${ }^{14}$ we also know that

$$
\frac{d K}{d t}+\frac{\left(I_{3}-I_{2}\right)}{I_{2} I_{3}} J_{2} J_{3}=0 .
$$

An approximation $I_{2} \approx I_{3}$ produces therefore an extra integral of motion $d K / d t=0$, which makes $K$ an adiabatic parameter. Yet, this additional integral of motion comes as a result of the rigid body approximation. The later may not be valid in the general case of vibrating molecule especially at the energies close to the dissociation limit when the geometry of the molecule is significantly distorted. The basic RRKM theory discriminates the cases when $K$ can be considered adiabatic and the cases when $K$ can be considered active, i.e., rapidly varying. The time scale of variation in $K$ for the case of molecules, such as ozone at energies near dissociation, is the subject of study in the present paper.

\section{SOFT BODY DYNAMICS}

As discussed in Sec. II, we are interested in the time dependence of the $K$ component of the total angular momentum of a triatomic molecule. We now allow the molecule to vibrate and thus consider its rotational dynamics as a softbody dynamics. We assume that at any given time the orientation of the principal axes of inertia of a triatomic molecule are known. Let us denote $F^{\prime}$ as the body-fixed frame of reference, i.e., the frame of reference in which the principal axes of inertia constitute orthogonal system of coordinates. We denote by $F$ a stationary reference frame. The direction of the total angular momentum $\mathbf{J}$ is fixed in the $F$ frame, while it changes in the $F^{\prime}$ frame. If the angular velocity of the frame $F^{\prime}$ with respect to $F$ is $\boldsymbol{\Omega}(t)$ then the evolution of the total angular momentum $d \mathbf{J} / d t$ in the frame $F$ is related to its evolution $d^{\prime} \mathbf{J} / d t$ in the frame $F^{\prime}$ as ${ }^{14}$

$$
\frac{d \mathbf{J}}{d t}=\frac{d^{\prime} \mathbf{J}}{d t}+\mathbf{\Omega} \times \mathbf{J} .
$$

Since $d \mathbf{J} / d t=0$, for the components of vector $\mathbf{J}=\left\{J_{1}, J_{2}, J_{3}\right\}$ we get

$$
\begin{aligned}
& \frac{d J_{1}}{d t}=\Omega_{3} J_{2}-\Omega_{2} J_{3}, \\
& \frac{d J_{2}}{d t}=\Omega_{1} J_{3}-\Omega_{3} J_{1}, \\
& \frac{d J_{3}}{d t}=\Omega_{2} J_{1}-\Omega_{1} J_{2} .
\end{aligned}
$$

Here we replaced $d^{\prime} / d t$ with $d / d t$ and from now on assume that differentiation with respect to time is given in the rotated $F^{\prime}$ frame.

The relation between the velocities $\mathbf{v}=d \mathbf{r} / d t$ and $\mathbf{v}^{\prime}$ $=d^{\prime} \mathbf{r} / d t$ of the atoms in the frames $F$ and $F^{\prime}$, respectively, has a form similar to Eq. (4),

$$
\mathbf{v}=\mathbf{v}^{\prime}+\boldsymbol{\Omega} \times \mathbf{r} .
$$

By definition, an angular momentum of a system of particles is $\mathbf{J}=\sum m \mathbf{r} \times \mathbf{v}$, which with Eq. (6) gives

$$
\mathbf{J}=\sum m \mathbf{r} \times \mathbf{\Omega} \times \mathbf{r}+\sum m \mathbf{r} \times \mathbf{v}^{\prime} .
$$

The first term in this expression is the angular momentum due to the rigid body rotation with an instantaneous geometry and instantaneous angular frequency of rotation. The second term represents the Coriolis coupling between the rotation of the $F^{\prime}$ frame and the vibrational motion within the $F^{\prime}$ frame. Given an instantaneous geometry of the molecule one can find the instantaneous principal axes, i.e., frame $F^{\prime}$, and the instantaneous principal inertia moments $I_{1}(t), I_{2}(t)$, and $I_{3}(t)$. With these principal inertia moments the first term in the right hand side of Eq. (7) is a vector with 
coordinates $\left\{I_{1}(t) \Omega_{1}(t), I_{2}(t) \Omega_{2}(t), I_{3}(t) \Omega_{3}(t)\right\}$, where $\Omega_{1}(t)$, $\Omega_{2}(t)$, and $\Omega_{3}(t)$ are the projections of $\boldsymbol{\Omega}(t)$ on the instantaneous principal axes of inertia [or in other words coordinates of $\boldsymbol{\Omega}(t)$ in $F^{\prime}$ frame].

If the coordinate of the center of mass is set at $\mathbf{r}=0$ then the second term in Eq. (7) is a vector orthogonal to the plane of the triatomic molecule, which means that Coriolis term contributes only to the $J_{3}$ projection of the angular momentum. We denote this contribution as $\delta J_{c} \equiv \sum m r v^{\prime} \sin \left(\widehat{r v^{\prime}}\right)$. The coordinates of the vector $\mathbf{J}$ in the $F^{\prime}$ frame are therefore

$$
\begin{aligned}
& J_{1}=I_{1} \Omega_{1}, \\
& J_{2}=I_{2} \Omega_{2}, \\
& J_{3}=I_{3} \Omega_{3}+\delta J_{c} .
\end{aligned}
$$

Expressing the instantaneous angular frequencies from Eq. (8) and substituting them into Eq. (5) we get

$$
\begin{aligned}
& \frac{d J_{1}}{d t}=\left(\frac{1}{I_{3}}-\frac{1}{I_{2}}\right) J_{2} J_{3}-\frac{\delta J_{c}}{I_{3}} J_{2}, \\
& \frac{d J_{2}}{d t}=\left(\frac{1}{I_{1}}-\frac{1}{I_{3}}\right) J_{1} J_{3}+\frac{\delta J_{c}}{I_{3}} J_{1}, \\
& \frac{d J_{3}}{d t}=\left(\frac{1}{I_{2}}-\frac{1}{I_{1}}\right) J_{1} J_{2} .
\end{aligned}
$$

These are equations for the "soft-body" rotational dynamics of a triatomic molecule. The evolution of $J_{1}, J_{2}$, and $J_{3}$ is modulated by the time dependent $I_{1}(t), I_{2}(t), I_{3}(t)$, and $\delta J_{c}(t)$. So far Eqs. (9a)-(9c) are exact. In chapters IV-VI we will provide an approximate solution to these equations. The main idea for the simplification of Eq. (9) is the possibility of separating the time scales of vibrations and rotations and thus treat variations in $I_{1}(t), I_{2}(t), I_{3}(t)$, and $\delta J_{c}(t)$ as a noise to the evolution of $J_{1}, J_{2}$, and $J_{3}$.

\section{DIFFERENTIAL EQUATION FOR $K(t)$}

Although $I_{1}, I_{2}$, and $I_{3}$ are functions of time, they vary around their equilibrium values $\left\langle I_{1}\right\rangle,\left\langle I_{2}\right\rangle$, and $\left\langle I_{3}\right\rangle$. For a near-symmetric top molecule such as ozone, where $\left\langle I_{1}\right\rangle:\left\langle I_{2}\right\rangle:\left\langle I_{3}\right\rangle=1: 9: 10$, we may neglect $1 / I_{2}$ and $1 / I_{3}$ with respect to $1 / I_{1}$ in Eqs. $(9 \mathrm{~b})$ and $(9 \mathrm{c})$. In that case we write

$$
\begin{aligned}
& \frac{d J_{2}}{d t}=\frac{J_{1}}{I_{1}} J_{3}+\frac{\delta J_{c}}{I_{3}} J_{1}, \\
& \frac{d J_{3}}{d t}=-\frac{J_{1}}{I_{1}} J_{2} .
\end{aligned}
$$

Omission of $I_{1}$ with respect to $I_{2}, I_{3}$ and equating $I_{2}$ with $I_{3}$ constitutes the basis of the symmetric top approximation. The present theory is therefore generally valid for any triatomic molecule rotational dynamics of which is known to be well described in the symmetric top approximation. Further in the paper we consider ozone molecule of $C_{2 v}$ symme- try as a particular example of such triatomic molecules. To simplify Eq. (9a) we note that for a flat molecule $I_{3}=I_{1}+I_{2}$ and thus we have

$$
\frac{d J_{1}}{d t}=-\left\{\left(\frac{I_{1}}{I_{2}}\right)\left(\frac{J_{3}}{I_{3}}\right)+\frac{\delta J_{c}}{I_{3}}\right\} J_{2} .
$$

The ratio $J_{3} / I_{3}$ is related to the $\xi_{3}$-projection of the rotational frequency, which is explicitly given by Eq. (8), thus

$$
\begin{aligned}
\frac{d J_{1}}{d t} & =-\left\{\left(\frac{I_{1}}{I_{2}}\right) \Omega_{3}+\left(1+\left(\frac{I_{1}}{I_{2}}\right)\right) \frac{\delta J_{c}}{I_{3}}\right\} J_{2} \\
& \approx-\left\{\left(\frac{I_{1}}{I_{2}}\right) \Omega_{3}+\frac{\delta J_{c}}{I_{3}}\right\} J_{2} .
\end{aligned}
$$

As we will show in section $\mathrm{V}$, the noise $\delta J_{c} / I_{3}$ for these molecules is of the order of the characteristic vibrational frequency $\Omega_{\text {vib }}$ of the molecule. Yet, $\Omega_{3}$ is of the order of the characteristic rotational frequency $\Omega_{\text {rot }}$. Typically rotational frequencies are much lower than the vibrational frequencies $\Omega_{\text {rot }} \ll \Omega_{\text {vib }}$. For a molecule such as ozone ${ }^{48} \mathrm{O}_{3}$, we can estimate $\Omega_{\text {rot }}$ that corresponds to the most probable angular momentum at room temperatures $J_{T}=\sqrt{k T I_{3}} \approx 17 \hbar$, i.e., $\Omega_{\text {rot }}$ $=J_{T} / I_{3} \approx 15 \mathrm{~cm}^{-1}$, which is 60 times smaller than the typical vibrational frequency of ozone $\sim 1000 \mathrm{~cm}^{-1}$. The coefficient $I_{1} / I_{2} \approx 0.1$ makes the first term in Eq. (12) even smaller; we therefore can omit the first term. The resulting simplified system of Eq. (9) for such molecules is thus

$$
\begin{aligned}
& \frac{d J_{1}}{d t}=-\frac{\delta J_{c}}{I_{3}} J_{2}, \\
& \frac{d J_{2}}{d t}=\frac{J_{1}}{I_{1}} J_{3}+\frac{\delta J_{c}}{I_{3}} J_{1}, \\
& \frac{d J_{3}}{d t}=-\frac{J_{1}}{I_{1}} J_{2} .
\end{aligned}
$$

One can check that Eqs. (13) conserve the total angular momentum, i.e., $(d / d t)\left(J_{1}^{2}+J_{2}^{2}+J_{3}^{2}\right)=0$. Since $J_{2}^{2}+J_{3}^{2}=J^{2}-K^{2}$ we search for solutions of $J_{2}$ and $J_{3}$ in the form

$$
\begin{aligned}
& J_{2}(t)=\sqrt{J^{2}-K(t)^{2}} \cos (\varphi(t)), \\
& J_{3}(t)=\sqrt{J^{2}-K(t)^{2}} \sin (\varphi(t)),
\end{aligned}
$$

where we introduced $K(t) \equiv J_{1}(t)$ and $\varphi(t)$ is some unknown function of time. It should be noted that the analytical solution for $J_{2}(t)$ and $J_{3}(t)$ for the general constant-energy rigid asymmetric top is expressed in terms of Jacobi elliptic functions $\operatorname{cn}(\tau)$ and $\operatorname{sn}(\tau) .{ }^{14}$ Yet, for the molecules with $I_{1} \ll I_{2}, I_{3}$ Jacobi elliptic functions transform into trigonometric functions $\operatorname{sn}(\tau) \approx \sin (\tau)$ and $\operatorname{cn}(\tau)=\cos (\tau)$, which physically correspond to the motion of a symmetric top. The latter approximation has already been incorporated in deriving Eq. (13). Substituting Eq. (14) into Eq. (13) we get

$$
\frac{d K}{d t}=-\frac{\delta J_{c}}{I_{3}} \sqrt{J^{2}-K^{2}} \cos \varphi,
$$




$$
\frac{d \varphi}{d t}=-\frac{K}{I_{1}}-\frac{\delta J_{c}}{I_{3}} \frac{K}{\sqrt{J^{2}-K^{2}}} \sin \varphi .
$$

We then integrate both parts of Eq. (15b) to yield

$$
\varphi(t)=\varphi_{0}-\int_{0}^{t} \frac{K}{I_{1}} d t^{\prime}-\int_{0}^{t} \frac{\delta J_{c}}{I_{3}} \frac{K}{\sqrt{J^{2}-K^{2}}} \sin \varphi d t^{\prime} .
$$

The Coriolis term $\delta J_{c}(t)$ rapidly oscillates about zero, while $K(t)$ is expected to change slowly (since in the rigid body symmetric top approximation it would have been constant) and their corresponding time scales are given in Sec. V. Therefore, the time integral of the first term in Eq. (15b) becomes much greater than the time integral of the second term in Eq. (15b) already after couple periods of oscillation of $\delta J_{c}(t)$. Equation (16) can be therefore solved iteratively by inserting it into itself and truncating it at the first order of $\int^{t} \delta J_{c}$, this iteration yields

$$
\begin{aligned}
\varphi(t)= & \left(-\int_{0}^{t} \frac{K}{I_{1}} d t^{\prime}+\varphi_{0}\right)-\int_{0}^{t} \frac{\delta J_{c}}{I_{3}} \frac{K}{\sqrt{J^{2}-K^{2}}} \\
& \times \sin \left(-\int_{0}^{t^{\prime}} \frac{K}{I_{1}} d t^{\prime \prime}+\varphi_{0}\right) d t^{\prime} .
\end{aligned}
$$

In expression (17), the first term is a slow and monotonic function of time, representing linear growth of phase $\varphi(t)$ with time, while the second term represents phase fluctuations. Preservation of phase fluctuation term is important since, as it will be shown below, it correlates with amplitude fluctuations resulting in additional drift coefficient. On substitution of the Eq. (17) into Eq. (15a) we get a selfconsistent equation for the $K$-projection of the angular momentum,

$$
\begin{aligned}
\frac{d K}{d t}= & -\frac{\delta J_{c}}{I_{3}} \sqrt{J^{2}-K^{2}} \cos \left(\int_{0}^{t} \frac{K}{I_{1}} d t^{\prime}-\varphi_{0}\right) \\
& -\frac{\delta J_{c}}{I_{3}} \sqrt{J^{2}-K^{2}} \sin \left(\int_{0}^{t} \frac{K}{I_{1}} d t^{\prime}-\varphi_{0}\right) \\
& \times \int_{0}^{t} \frac{\delta J_{c}}{I_{3}} \frac{K}{\sqrt{J^{2}-K^{2}}} \sin \left(\int_{0}^{t^{\prime}} \frac{K}{I_{1}} d t^{\prime \prime}-\varphi_{0}\right) d t^{\prime},
\end{aligned}
$$

where we have also used the Taylor expansion $\cos \left(x_{0}+\Delta x\right)$ $=\cos \left(x_{0}\right)-\sin \left(x_{0}\right) \Delta x$, with $\Delta x$ being the phase fluctuation term in Eq. (17). This equation is the central result of the present section. Given the time dependence of the vibrational variation in $I_{1}(t), I_{3}(t)$, and $\delta J_{c}(t)$, it provides an explicit expression for the dynamics of the $K$-projection of angular momentum. One can see that since $d K / d t$ is proportional to the Coriolis term $\delta J_{c}(t)$, the dynamics of $K(t)$ is governed by Coriolis coupling.

\section{CHARACTERISTIC TIME SCALES}

Before we further simplify Eq. (18) let us consider the characteristic time scales of the parameters $I_{1}(t), I_{3}(t), \delta J_{c}(t)$, and $K(t)$ in Eq. (18). We again consider ozone molecule as an example.
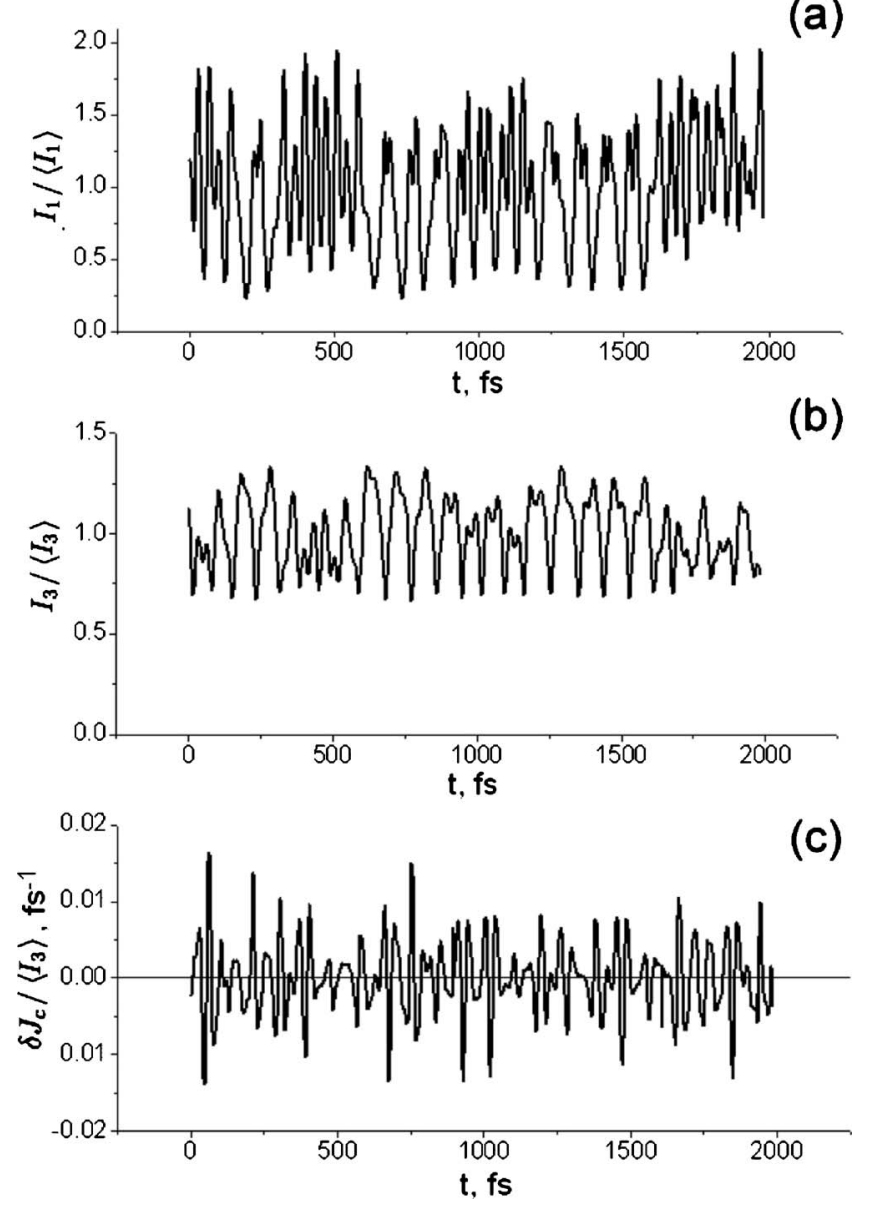

FIG. 2. Characteristic dynamics of (a) $I_{1}(t)$, (b) $I_{3}(t)$, and (c) $\delta J_{c}(t)$.

The variation in the principal moments of inertia $I_{1}(t)$ and $I_{3}(t)$ is governed by vibrational dynamics of a triatomic molecule. At the energies of dissociation, both $I_{1}(t)$ and $I_{3}(t)$ show oscillatory dynamics around their equilibrium values with randomly varying frequencies and amplitudes, as in Fig. 2 , given for an ozone molecule. One can see that the frequency of oscillations of $I_{1}(t)$ randomly switches between the bending oscillation frequency at $\omega_{b} \approx 700 \mathrm{~cm}^{-1}$ and the normal mode stretching frequency at $\omega_{s} \approx 1100 \mathrm{~cm}^{-1}$ due to vibrational chaos. The instantaneous equilibrium of $I_{1}(t)$, as well as the amplitude of its oscillations, depends on the instantaneous geometry of the ozone molecule and its instantaneous dynamical regime. Since $I_{1}(t)$ exhibits random oscillations with the characteristic time scale $T_{b}=2 \pi / \omega_{b} \approx 60 \mathrm{fs}$, we can treat $I_{1}$ on the time scale $t \gg T_{b}$ as a random variable. The same analysis is valid for the variation in $I_{3}(t)$, except that it mainly participates in oscillations with bending frequency $\omega_{b}$, since normal mode stretching oscillations do not significantly change the moment of inertia around the axis orthogonal to the plane of ozone molecule.

We now consider the variation in the most important term, Coriolis term, $\delta J_{c}(t)$. As one can see from Fig. 2, this quantity is the only variable that changes its sign. The origin of Coriolis term $\delta J_{c}(t)$ is illustrated in Fig. 3: it is an instantaneous "internal" angular momentum of ozone molecule with respect to the principal axes of inertia. It is given by the explicit expression (see notations in Fig. 4) 


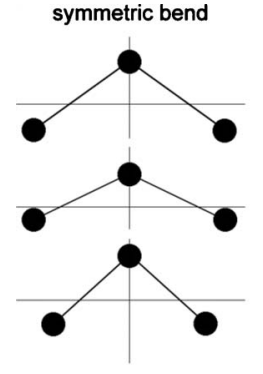

$\delta J_{c}(t)=0$

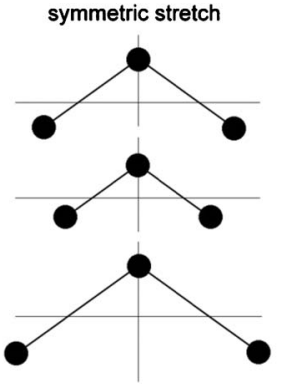

$\delta J_{c}(t)=0$

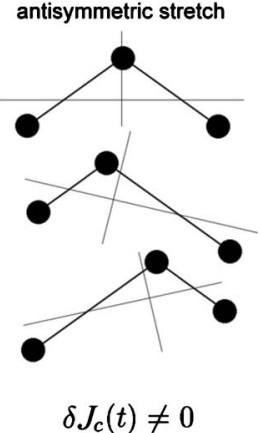

$\delta J_{c}(t) \neq 0$

FIG. 3. Coriolis angular momentum term $\delta J_{c}$ for different types of vibrational modes in isotopically symmetric ozone molecule. Orthogonal lines represent $\xi_{1}$ and $\xi_{2}$ principal axes of inertia.

$$
\delta J_{c}=\sum m \mathbf{r} \times \mathbf{v}^{\prime}=m_{1} r_{1}^{2} \frac{d \varphi_{1}}{d t}+m_{2} r_{2}^{2} \frac{d \varphi_{2}}{d t}+m_{3} r_{3}^{2} \frac{d \varphi_{3}}{d t} .
$$

The Coriolis term $\delta J_{c}$ does not appear in symmetric oscillations of isotopically symmetric molecules; we discuss this in detail in Sec. IX. For the purpose of simplicity, in the present paper we consider only an isotopically symmetric ozone molecule ${ }^{48} \mathrm{O}_{3}$ and therefore the characteristic time scale of $\delta J_{c}(t)$ is that of the antisymmetric normal mode with the frequency $d \varphi_{i} / d t \approx \omega_{\text {asymm }} \approx 1100 \mathrm{~cm}^{-1}$, i.e., $30 \mathrm{fs}$. At time steps $\Delta t>30$ fs one can therefore consider $\delta J_{c}$ as a random variable evenly distributed around zero.

Yet, the change in $K(t)$ is much slower. Indeed, the characteristic time scale of any dynamical physical parameter is inversely proportional to the strength of its driving force. Obviously, the interatomic forces in ozone molecule are much stronger than the strength of Coriolis coupling. The driving forces for $I_{1}(t), I_{2}(t)$, and $I_{3}(t)$ are interatomic forces, while the driving force for $K(t)$ is the Coriolis coupling, as seen from Eq. (18). This result leads to the conclusion that the characteristic time scale $T_{K}$ of $K(t)$ should be much greater than the characteristic time scales $T_{I} \approx 60$ fs of $I_{1}(t)$, $I_{2}(t)$, and $I_{3}(t)$. This result is confirmed by numerical simulations shown in Fig. 5, giving $T_{K} \sim 1000 T_{I}$.

The analysis of the present section suggests that we can treat vibrational motion of ozone molecule as a random noise in the evolution of $K(t)$ due to the presence of vibrational chaos at high vibrational energies and due to the difference in characteristic time scales of $K(t)$ and $I_{1}(t), I_{3}(t)$, and

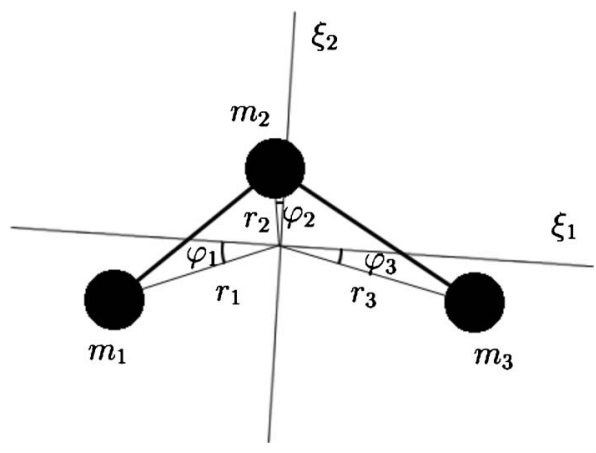

FIG. 4. Ozone molecule in the $\xi_{1}-\xi_{2}$ plane.

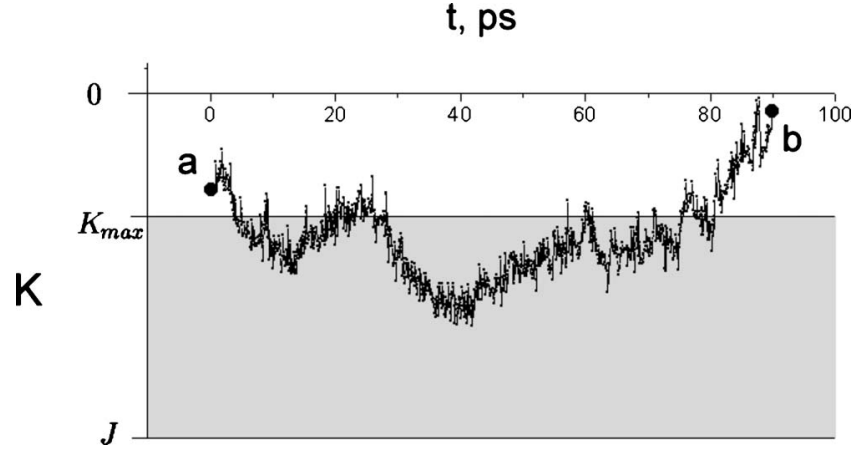

FIG. 5. An effective diffusion of $K$-projection of rotational angular momentum in ozone molecule. The figure represents a single trajectory of $K(t)$ from the MD simulation of a molecule of ozone. Point (a) corresponds to the formation of ozone molecule and point (b) corresponds to the dissociation of ozone molecule. The dark region corresponds to the energies at which ozone molecule cannot dissociate (closed states); the white region corresponds to open states.

$\delta J_{c}(t)$. Under the influence of such a noise [given by Eq. (18)] $K(t)$ exhibits a diffusionlike (Brownian) motion shown in Fig. 5. This result leads us to the conclusion that Eq. (18) can be equivalent to some sort of diffusion equation, the form of which is obtained in Sec. VI.

\section{EFFECTIVE DIFFUSION EQUATION FOR $K(t)$}

In Sec. IV we obtained the differential equation for $K(t)$ in the form

$$
\frac{d K}{d t}=V(t)+U(t)
$$

where $V(t)$ and $U(t)$ are rapidly fluctuating terms,

$$
\begin{aligned}
V(t)= & -\frac{\delta J_{c}}{I_{3}} \sqrt{J^{2}-K^{2}} \cos \left(\int_{0}^{t} \frac{K}{I_{1}} d t^{\prime}-\varphi_{0}\right) \\
U(t)= & -\frac{\delta J_{c}}{I_{3}} \sqrt{J^{2}-K^{2}} \sin \left(\int_{0}^{t} \frac{K}{I_{1}} d t^{\prime}-\varphi_{0}\right) \\
& \times \int_{0}^{t} \frac{\delta J_{c}}{I_{3}} \frac{K}{\sqrt{J^{2}-K^{2}}} \sin \left(\int_{0}^{t^{\prime}} \frac{K}{I_{1}} d t^{\prime \prime}-\varphi_{0}\right) d t^{\prime} .
\end{aligned}
$$

Since $U(t)$ contains an integral over the sign-alternating function $\delta J_{c}(t)$ we expect that $V(t)$ plays dominant effect on the time evolution of $K(t)$, while $U(t)$ contributes minor correction. While both $V$ and $U$ terms are treated by us explicitly in this section, we found it simpler to present analysis first for the major $V(t)$ term and then to introduce the $U(t)$ term into the discussion. Therefore, in the first part of this section, we analyze the effect of the $V(t)$ term on the evolution of $K(t)$, while in the second part we analyze the effect of both $V(t)$ and $U(t)$ terms on $K(t)$. Yet, the main physics is captured by the $V(t)$ term and the next section reveals the central idea of the paper.

\section{A. Diffusion of $K$ under the influence of $V(t)$}

Let us find how $K(t)$ behaves under the action of the $V(t)$ term, which is the dominant term in Eq. (20), 


$$
\frac{d K}{d t}=V(t)
$$

The right side of Eq. (22) serves as a generalized velocity of $K(t)$. Since $V$ rapidly and randomly changes, Eq. (22) is explicitly an equation for an overdamped one-dimensional Brownian motion with coordinate $K$. The key quantity that describes Brownian motion is its velocity-velocity correlation function

$$
C_{V}(t)=\langle V(t) V(0)\rangle
$$

Below we study $C_{V}(t)$ in detail. As discussed in Sec. V, since the time scale of $K(t)$ is three orders of magnitude greater than the time scales of $I_{1}(t), I_{2}(t)$, and $\delta J_{c}(t)$, the average over $K(t)$ in Eq. (23) can be taken independently of the averages over $I_{1}(t), I_{2}(t)$, and $\delta J_{c}(t)$. The parameter $I_{3}(t)$ can be considered independent of $\delta J_{c}(t)$ since the fluctuations of the former mostly depend on the bending oscillation (as discussed in Sec. V) and the fluctuations of the latter mostly depend on the antisymmetric stretch oscillation. Finally, the characteristic time scale of the variable $\cos \left(\int^{t}\left(K / I_{1}\right) d t^{\prime}\right)$, due to the presence of integral, is $2 \pi /\left(K /\left\langle I_{1}\right\rangle\right)$, which is approximately a characteristic period of ozone rotation about the $\xi_{1}$ principal axis of inertia. In Sec. $\mathrm{V}$ we estimated this period to be one order of magnitude larger than the vibrational period and therefore two orders of magnitude smaller than the characteristic time of $K(t)$; therefore $\cos \left(\int^{t}\left(K / I_{1}\right) d t^{\prime}\right)$ can be considered independent of other terms such as $K(t)$ and $I_{3}(t)$, $\delta J_{c}(t)$ in taking the average in Eq. (23). These simple considerations greatly simplify expression (23),

$$
\begin{aligned}
C_{V}(t)= & \left(J^{2}-K(0)^{2}\right) \frac{1}{\left\langle I_{3}\right\rangle^{2}}\left\langle\delta J_{c}(t) \delta J_{c}(0)\right\rangle \\
& \times\left\langle\cos \left(\int_{0}^{t} \frac{K}{I_{1}} d t^{\prime}-\varphi_{0}\right) \cos \left(\varphi_{0}\right)\right\rangle .
\end{aligned}
$$

Here we wrote $\left(J^{2}-K(0)^{2}\right)$ instead of its correlation function $\left\langle\sqrt{J^{2}-K^{2}(t)} \sqrt{J^{2}-K(0)^{2}}\right\rangle$ since obviously it is a constant on the time scale of the correlation time of $\left\langle\delta J_{c}(t) \delta J_{c}(0)\right\rangle$; we also simplified $\left\langle 1 / I_{3}(t) I_{3}(0)\right\rangle=\left\langle 1 /\left(\left\langle I_{3}\right\rangle+\Delta I_{3}(t)\right)\left(\left\langle I_{3}\right\rangle\right.\right.$ $\left.\left.+\Delta I_{3}(0)\right)\right\rangle=\left(1 /\left\langle I_{3}\right\rangle^{2}\right)-\left(\left\langle\Delta I_{3}(t)\right\rangle /\left\langle I_{3}\right\rangle^{3}\right)-\left(\left\langle\Delta I_{3}(0)\right\rangle /\left\langle I_{3}\right\rangle^{3}\right)$ $=1 /\left\langle I_{3}\right\rangle^{2}, \Delta I_{i}(t)=I_{i}-\left\langle I_{i}\right\rangle$. Performing averaging over the initial phase $\varphi_{0}$ in the last term of Eq. (24) we simplify $C_{V}(t)$ further

$$
C_{V}(t)=\frac{\left(J^{2}-K(0)^{2}\right)}{2\left\langle I_{3}\right\rangle^{2}}\left\langle\delta J_{c}(t) \delta J_{c}(0)\right\rangle\left\langle\cos \left(\int_{0}^{t} \frac{K}{I_{1}} d t^{\prime}\right)\right\rangle
$$

The correlation time scale of $\left\langle\delta J_{c}(t) \delta J_{c}(0)\right\rangle$ is expected, for ozone, to be of the order a few vibrational periods of the molecule due to the vibrational chaos at the energies near dissociation, while the time scale of a characteristic oscillation of $\left\langle\cos \left(\int_{0}^{t}\left(K / I_{1}\right) d t^{\prime}\right)\right\rangle$ (rotational period), as discussed above, is one order of magnitude greater than that, therefore
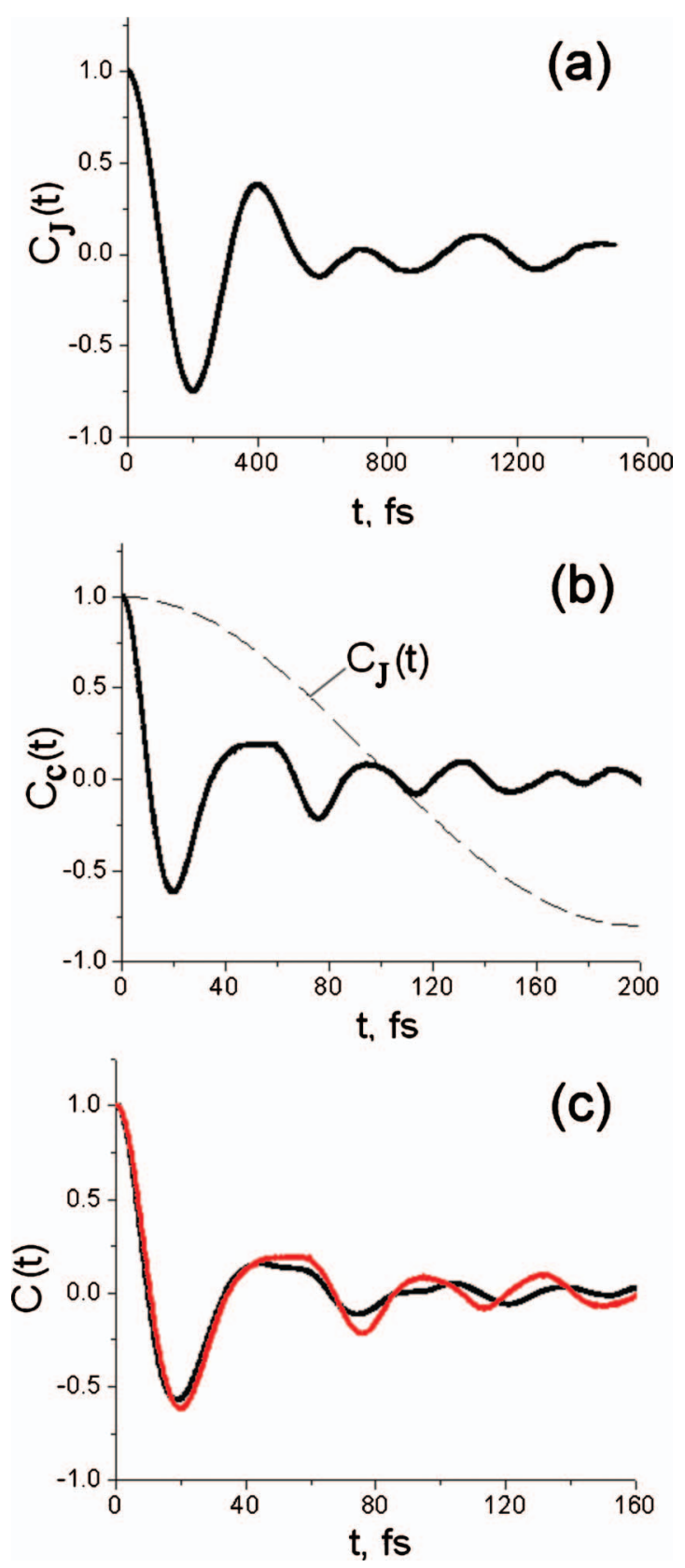

FIG. 6. Correlation functions governed by vibrational chaos: (a) normalized correlation function $C_{J}(t)=\left\langle J_{2}(t) J_{2}(0)\right\rangle$, (b) normalized correlation function $C_{c}(t)=\left\langle\delta J_{c}(t) \delta J_{c}(0)\right\rangle$, and (c) normalized correlation function $C(t)$ $=\left\langle J_{2}(t) \delta J_{c}(t) J_{2}(0) \delta J_{c}(0)\right\rangle$ (black line) compared to $C_{c}(t)$ (color line).

the decay of the whole correlation function $C_{V}(t)$ is defined by the decay of $\left\langle\delta J_{c}(t) \delta J_{c}(0)\right\rangle$. The latter is illustrated in Fig. 6. Interestingly enough, the correlation time of $C_{V}(t)$ is of the order of several vibrational periods, which in terms of physical numbers constitutes $t_{\text {corr }} \approx 0.1 \mathrm{ps}$. Yet, the mean life time of ozone molecule at room temperatures obtained from experiment is $200 \mathrm{ps}$, which means that intramolecular dynamics occurs on the time scale much larger than the correlation time $t_{\text {corr }}$ of $C_{V}(t)$. At these times the effect of noise $V_{K}(t)$ will be the same as if the noise was delta correlated with the correlation function $\widetilde{\delta(t)}$ defined as $2 \int_{0}^{\infty} \widetilde{\delta(t)} d t=\int_{0}^{\infty} C_{V}(t) d t .{ }^{15}$ The last integral stands for the definition of the diffusion coefficient $D$ of a Brownian motion, ${ }^{15}$ 


$$
D=\int_{0}^{\infty} C_{V}(t) d t
$$

We therefore arrive at the conclusion that during the lifetime of the ozone molecule the behavior of $K(t)$ can be described by a simple diffusion law

$$
\frac{d K}{d t}=\sqrt{D} \xi(t),
$$

with

$$
\langle\xi(t) \xi(0)\rangle=2 \delta(t) .
$$

The only remaining step is to find the expression for the diffusion coefficient $D$. Substituting Eq. (25) into Eq. (26) we get

$$
D=\frac{\left(J^{2}-K(0)^{2}\right)}{2\left\langle I_{3}\right\rangle^{2}} \int_{0}^{\infty}\left\langle\delta J_{c}(t) \delta J_{c}(0)\right\rangle\left\langle\cos \left(\int_{0}^{t} \frac{K}{I_{1}} d t^{\prime}\right)\right\rangle d t .
$$

For any two functions $f(t), g(t)$ such that $f(t)$ decays to zero much faster than $g(t)$ we can write $\int_{0}^{\infty} f(t) g(t) d t$ $\approx g(0) \int_{0}^{\infty} f(t) d t$. Equation (29) can be thus simplified,

$$
D=\frac{\left(J^{2}-K(0)^{2}\right)}{2\left\langle I_{3}\right\rangle^{2}} \int_{0}^{\infty}\left\langle\delta J_{c}(t) \delta J_{c}(0)\right\rangle d t .
$$

This is the final expression for the diffusion coefficient. One can see that the diffusion coefficient depends on the correlation function $\left\langle\delta J_{c}(t) \delta J_{c}(0)\right\rangle$, which behavior is defined by the vibrational chaos. We cannot treat vibrational chaos analytically. Yet, a great simplification comes from the fact this correlation function depends only on vibrational energy $E_{\mathrm{vib}}$, therefore any combination of the total energy $E$ of the molecule and the rotational energy $E_{\text {rot }}$ of the molecule that result in the same vibrational energy, i.e., $E_{\mathrm{vib}}=E-E_{\mathrm{rot}}=$ const, should give the same value of $\int_{0}^{\infty}\left\langle\delta J_{c}(t) \delta J_{c}(0)\right\rangle d t$. We therefore define $D_{c}\left(E_{\mathrm{vib}}\right) \equiv\left(1 / 2\left\langle I_{3}\right\rangle^{2}\right) \int_{0}^{\infty}\left\langle\delta J_{c}(t) \delta J_{c}(0)\right\rangle d t$ and find $D_{c}(x)$ numerically. This calculation is given in Sec. X, where the numerical simulations are discussed. It is found that $D_{c}\left(E_{\mathrm{vib}}\right)$ is a very slow function of $E_{\mathrm{vib}}$ in the range of vibrational energies of interest near the dissociation limit. Combining Eqs. (27) and (30) we arrive at the stochastic equation that describes the dynamics of $K(t)$ in the molecule of a total energy $E$ and total angular momentum $J$ on the time scale $t \gg t_{c}$, where $t_{c}$ is $0.1 \mathrm{ps}$ for ozone,

$$
\frac{d K}{d t}=\sqrt{J^{2}-K^{2}} \sqrt{D_{c}\left(E_{\mathrm{vib}}\right)} \xi(t),
$$

where

$$
E_{\mathrm{vib}}(K)=E-\frac{J^{2}}{2 \sqrt{\left\langle I_{2}\right\rangle\left\langle I_{3}\right\rangle}}-\left(\frac{1}{\left\langle I_{1}\right\rangle}-\frac{1}{\sqrt{\left\langle I_{2}\right\rangle\left\langle I_{3}\right\rangle}}\right) \frac{K^{2}}{2},
$$

$\xi(t)$ is a standard delta-correlated noise $\langle\xi(t) \xi(0)\rangle=2 \delta(t)$ and $D_{c}(x)$ is given by Eq. (43). In the derivation of Eq. (31) we did not introduce any special differentiation rules, i.e., no Ito rules, and so the stochastic Eq. (31) should be understood in the Stratanovich form. ${ }^{15}$
The $K$-dependence of the diffusion coefficient in Eq. (31) has an interesting effect: while the slope of the average $\left\langle(K-K(0))^{2}\right\rangle$ is always positive, the slope of $\left\langle K^{2}-K(0)^{2}\right\rangle$ may be positive as well as negative. In particular, this behavior of $\left\langle K^{2}-K(0)^{2}\right\rangle$ has been observed in numerical simulations on vibrational-rotational intramolecular energy exchange in ozone in Ref. 8. In Fig. 2 of the latter paper ${ }^{8}$ the change in rotational energy with time $\Delta E_{\mathrm{rot}}(t)$ has a linear form on the time scale of $400 \mathrm{ps}$ with positive or negative slopes depending on the values of $K(0)$. In the Appendix we show that this behavior follows directly from the solution of the Eq. (31). Indeed, since the rotational energy is proportional to $K^{2}(t)$ [as in Eq. (2)], it follows from the Appendix that $\left\langle\Delta E_{\mathrm{rot}}(t)\right\rangle \sim\left\langle K(t)^{2}-K(0)^{2}\right\rangle \sim\left(J^{2}-2 K(0)^{2}\right) t$. For the values of $K(0)$ such that $K(0)<J / \sqrt{2}$ the slope of $\left\langle\Delta E_{\text {rot }}(t)\right\rangle$ is positive, while for $K(0)>J / \sqrt{2}$ it is negative. These results are in agreement with the observed numerical results in Ref. 8 as well as the critical value of $K(0)$ at which the slope of $\left\langle\Delta E_{\text {rot }}(t)\right\rangle$ changes its sign. For $J=20$ this critical value is $J / \sqrt{2}=14$, while in Ref. 8 it lies somewhere between $K(0)$ $=10$ and $K(0)=14$. The slight difference between the "crude" analytical value of $K(0)=J / \sqrt{2}$ and the results of MD simulations is due to the dependence of $D_{c}$ on $E_{\text {vib }}$ (while in the Appendix we assumed it constant) and due to the additional drift coefficient induced by the $U(t)$ term in Eq. (20), which we discuss in Sec. VI B.

\section{B. Diffusion of $K$ under the influence of both $V(t)$ and $U(t)$}

We now include the second term, $U(t)$, in our discussion. While in general its contribution to the behavior of $K(t)$ is small, it becomes dominant when the effect of the $V(t)$ term becomes small or zero: in particular, at points $K= \pm J$, at which Eq. (31) gives zero diffusion coefficient.

Using the same arguments as in Sec. VI A, we can easily find the first two moments of $U(t)$,

$$
\begin{aligned}
\langle U(t)\rangle= & -\frac{1}{\left\langle I_{3}\right\rangle^{2}} \int_{0}^{t} d t^{\prime} K\left(t^{\prime}\right)\left\langle\delta J_{c}(t) \delta J_{c}\left(t^{\prime}\right)\right\rangle \\
& \times\left\langle\sin \left(\int_{0}^{t} \frac{K}{I_{1}} d t^{\prime}-\varphi_{0}\right) \sin \left(\int_{0}^{t^{\prime}} \frac{K}{I_{1}} d t^{\prime \prime}-\varphi_{0}\right)\right\rangle_{\varphi_{0}} \\
= & -\frac{K(t)}{2\left\langle I_{3}\right\rangle^{2}} \int_{0}^{t}\left\langle\delta J_{c}(\tau) \delta J_{c}(0)\right\rangle d \tau=-K D_{c},
\end{aligned}
$$

where we have used the property of invariance of correlation function under the time shift, i.e., $\left\langle\delta J_{c}(t+\tau) \delta J_{c}(t)\right\rangle$ $=\left\langle\delta J_{c}(\tau) \delta J_{c}(0)\right\rangle$, and the subscript $\varphi_{0}$ denotes averaging over initial phases $\varphi_{0}$. One can see that $U(t)$ contributes additional drift coefficient of $-D_{c} K$ to the evolution of $K(t)$, which has maximum effect at $K= \pm J$.

To find the second moment of $U(t)$ we first derive an expression for $\int_{0}^{t} d t_{1} \int_{0}^{t} d t_{2}\left\langle U\left(t_{1}\right) U\left(t_{2}\right)\right\rangle$. For the four-point average of $\delta J_{c}$ we may use the approximate relation $\left\langle x_{1} x_{2} x_{3} x_{4}\right\rangle=\left\langle x_{1} x_{2}\right\rangle\left\langle x_{3} x_{4}\right\rangle+\left\langle x_{1} x_{3}\right\rangle\left\langle x_{2} x_{4}\right\rangle+\left\langle x_{1} x_{4}\right\rangle\left\langle x_{2} x_{3}\right\rangle$, in the case of the Gaussian stochastic process this approximation is exact. ${ }^{15}$ We thus have 

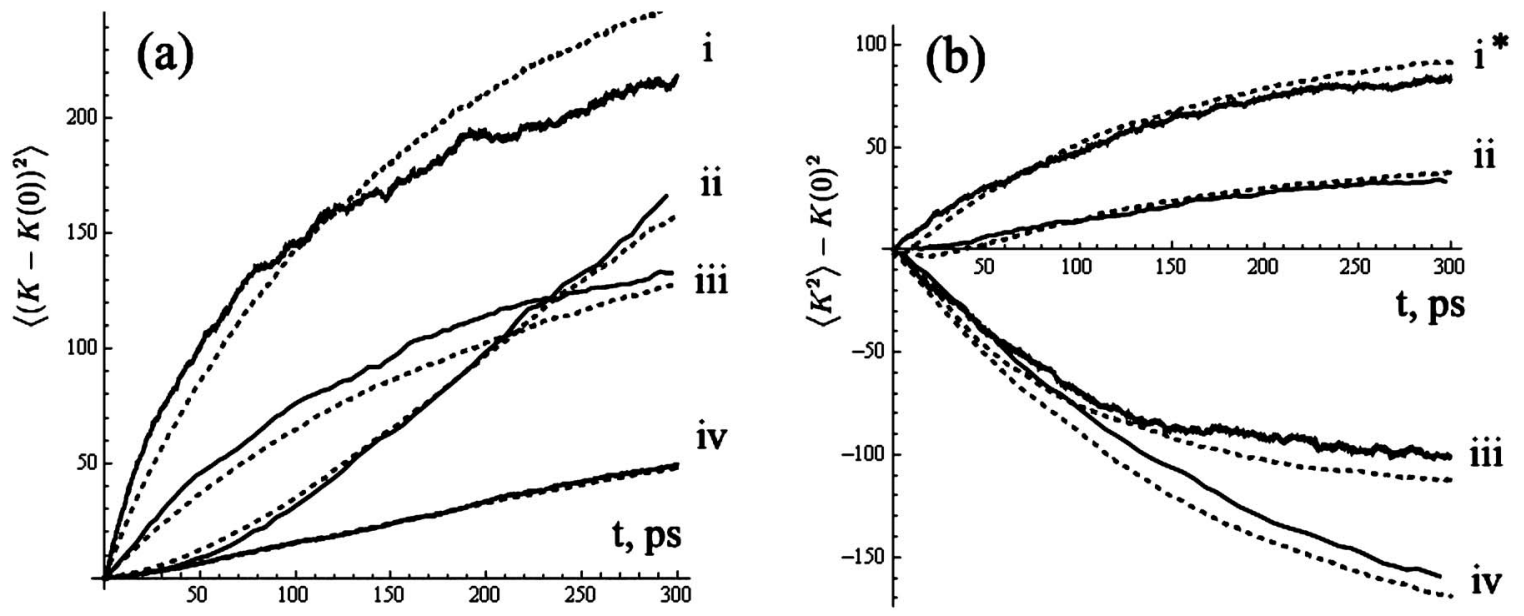

FIG. 7. Long-time behavior of mean squared value and mean square deviation of $K$. Solid lines represent results of MD simulations for ozone molecule with total angular momentum $J$, initial value of $K$-projection $K(0)$ and total energy $E$ in eV; dashed lines are obtained from integration of stochastic Eq. (36) for the corresponding parameters $J, K(0)$, and $E$. Inset (a): (i) $J=30, K(0)=8$, and $E=-0.01$; (ii) $J=20, K(0)=18$, and $E=-0.025$; (iii) $J=20, K(0)=8$, and $E=-0.025$; (iv) $J=10, K(0)=8$, and $E=-0.01$. Inset (b): $J=20$ and $E=-0.025$; (i) $K(0)=4$; (ii) $K(0)=8$; (iii) $K(0)=15$; (iv) $K(0)=18$. The star indicates that correction (41) has been used in case (b)(i).

$$
\begin{aligned}
\int_{0}^{t} d t_{1} \int_{0}^{t} d t_{2}\left\langle U\left(t_{1}\right) U\left(t_{2}\right)\right\rangle= & \frac{1}{\left\langle I_{3}\right\rangle^{4}} \frac{1}{4} \int_{0}^{t} d t_{1} \int_{0}^{t} d t_{2} \int_{0}^{t} d t^{\prime} \int_{0}^{t} d t^{\prime \prime} \frac{\sqrt{J^{2}-K\left(t_{1}\right)^{2}}}{\sqrt{J^{2}-K\left(t^{\prime}\right)^{2}}} K\left(t^{\prime}\right) \frac{\sqrt{J^{2}-K\left(t_{2}\right)^{2}}}{\sqrt{J^{2}-K\left(t^{\prime \prime}\right)^{2}}} K\left(t^{\prime \prime}\right)\left\{\left\langle\delta J_{c}\left(t_{1}\right) \delta J_{c}\left(t_{2}\right)\right\rangle\right. \\
& \left.\times\left\langle\delta J_{c}\left(t^{\prime}\right) \delta J_{c}\left(t^{\prime \prime}\right)\right\rangle+\left\langle\delta J_{c}\left(t_{1}\right) \delta J_{c}\left(t^{\prime}\right)\right\rangle\left\langle\delta J_{c}\left(t_{2}\right) \delta J_{c}\left(t^{\prime \prime}\right)\right\rangle+\left\langle\delta J_{c}\left(t_{1}\right) \delta J_{c}\left(t^{\prime \prime}\right)\right\rangle\left\langle\delta J_{c}\left(t_{2}\right) \delta J_{c}\left(t^{\prime}\right)\right\rangle\right\} \\
= & \int_{0}^{t} d t_{1} \int_{0}^{t} d t^{\prime}\left(\frac{J^{2}-K\left(t_{1}\right)^{2}}{J^{2}-K\left(t^{\prime}\right)^{2}}\right) K\left(t^{\prime}\right)^{2} D_{c}\left(t_{1}\right) D_{c}\left(t^{\prime}\right)+\int_{0}^{t} d t_{1} \int_{0}^{t} d t_{2} K\left(t_{1}\right) K\left(t_{2}\right) D_{c}\left(t_{1}\right) D_{c}\left(t_{2}\right) \\
& +\int_{0}^{t} d t_{1} \int_{0}^{t} d t_{2} K\left(t_{1}\right) K\left(t_{2}\right) D_{c}\left(t_{1}\right) D_{c}\left(t_{2}\right) \approx 3 \int_{0}^{t} d t_{1} \int_{0}^{t} d t_{2}\left(K\left(t_{1}\right) D_{c}\left(t_{1}\right)\right)\left(K\left(t_{2}\right) D_{c}\left(t_{2}\right)\right)
\end{aligned}
$$

From Eq. (34) one can see that the second moment of $U(t)$ is

$$
\left\langle U(t)^{2}\right\rangle=3 K(t)^{2} D_{c}(t)^{2} .
$$

One can see from Eq. (34) that since $K(t)$ and $D_{c}(K(t))$ are slow functions of time then the average $\left\langle\left(\int^{t} U\left(t^{\prime}\right) d t^{\prime}\right)^{2}\right\rangle$ grows as $O\left(t^{2}\right)$, while from Eq. (33) it follows that the average $\left\langle\int^{t} U\left(t^{\prime}\right) d t^{\prime}\right\rangle$ grows as $O(t)$. The effect of $U(t)$ on the behavior of $K(t)$ is then similar to an additional "constant" term, which gives linear growth in time after a single integration over time and quadratic growth after double integration over time. We can then effectively replace $U(t)$ in the Eq. (20) with the "noiseless" term $\tilde{U}(t) \equiv-\sqrt{2} D_{c} K$, which has similar properties to the original $U(t)$ term, i.e., $\langle\tilde{U}\rangle=-\sqrt{2} D_{c} K$ $\approx-D_{c} K=\langle U\rangle$ from Eq. (33)] and $\left\langle\tilde{U}^{2}\right\rangle=2 D_{c}^{2} K^{2} \approx 3 D_{c}^{2} K^{2}$ $=\left\langle U^{2}\right\rangle$ [from Eq. (35)]. Combining Eq. (20) with Eq. (31) and the results of the present section, the final stochastic equation for diffusion of $K(t)$ under the influence of both $V(t)$ and $U(t)$ terms then reads

$$
\frac{d K}{d t}=-\sqrt{2} D_{c} K+\sqrt{J^{2}-K^{2}} \sqrt{D_{c}} \xi(t) .
$$

Equation (36) is the central result of the present paper. Its short and long time behavior is tested in the numerical Sec.
$\mathrm{X}$ and one observes good agreement with the direct simulations of vibrationally excited ozone molecule, as in Fig. 7. The reader may proceed directly to Sec. VIII without loss of understanding, skipping the discussion on corrections to symmetric top approximation presented in Sec. VII.

\section{CORRECTION TO THE SYMMETRIC TOP APPROXIMATION}

In Secs. II-VI we discussed the effect of vibrations on rotational dynamics of a triatomic molecule using the nearsymmetric top approximation, i.e., assuming $I_{2}=I_{3}$. As can be seen in Fig. 8, the symmetric top approximation works very well for most values of $K$, except for very small ones. For small values of $K$, the rotational dynamics of a triatomic molecule can be trapped in the region where the vector of the total angular momentum $\mathbf{J}$ rotates (in the body-fixed frame), not around the $\xi_{1}$ axis but around the $\xi_{3}$ axis, as in the closed trajectory around $\xi_{3}$ axis in Fig. 8. These rotational dynamics are well known since for a general rigid body, there are two stable axes of rotation: ${ }^{14}$ one which corresponds to the minimum moment of inertia and the other which corresponds to the maximum moment of inertia. The separatrix between the regions of stable rotations is given in parametric form, ${ }^{14}$ 


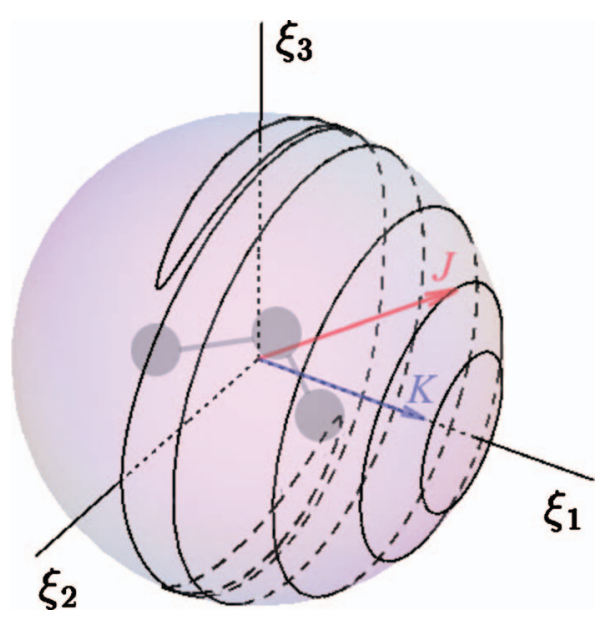

FIG. 8. Rotation of total angular momentum $\mathbf{J}$ in the body-fixed frame of a rigid triatomic molecule. The sphere $J_{1}^{2}+J_{2}^{2}+J_{3}^{2}=J^{2}$ represents all possible orientations of the total angular momentum $\mathbf{J}$. Every closed trajectory on the sphere is formed by the end points of rotating $\mathbf{J}$ at some rotational energy $E_{\text {rot }}$, i.e., the closed trajectories are the intersections of the sphere $J_{1}^{2}+J_{2}^{2}$ $+J_{3}^{2}=J^{2}$ with the ellipsoid $\left(J_{1}^{2} / 2 I_{1}\right)+\left(J_{2}^{2} / 2 I_{2}\right)+\left(J_{3}^{2} / 2 I_{3}\right)=E_{\text {rot }}$

$$
\begin{aligned}
& J_{1}^{s x}= \pm J \frac{I_{1}}{I_{2}} \frac{1}{\cosh (\tau)}, \\
& J_{2}^{s x}=J \tanh (\tau), \\
& J_{3}^{s x}= \pm J \sqrt{\frac{I_{3}}{I_{2}}\left(1-\frac{I_{1}}{I_{2}}\right)} \frac{1}{\cosh (\tau)},
\end{aligned}
$$

where parameter $\tau$ parametrizes three-dimensional curve (37) and goes from $-\infty$ to $\infty$. For values of $K$, such that $|K|$ $>\left(I_{1} / I_{2}\right) J$ vector $\mathbf{J}$ lies outside the separatrix region (37) and therefore rotates only around $\xi_{1}$-axis, while for $|K|$ $<\left(I_{1} / I_{2}\right) J$ it can also rotate around $\xi_{3}$-axis. ${ }^{14}$ The area of stable rotation around $\xi_{3}$-axis scales as $\left(I_{1} / I_{2}\right) J$ and is very small for molecules with $I_{1} \ll I_{2}$, such as ozone. Yet, in this region, $K$ is not a constant of motion any more and oscillates around zero value with a frequency of molecular rotation around $\xi_{3}$-axis, which is of the order of $J / I_{3}$. In the present section we discuss the effects of the latter fast oscillations of $K$ on the general model of diffusion (36).

Under the influence of vibrational chaos, by means of the Coriolis coupling, vector $\mathbf{J}$ may jump from one region of stable rotation to the other, i.e., may jump over the separatrix boundary (37) back and forth, as in Fig. 9. The theoretical description of this behavior is very difficult and requires analysis of noise-induced transitions in a nonlinear system with bifurcations. ${ }^{16}$ In the present section we provide a simplified approximate treatment of the problem and estimate the effect of additional domain of stable rotations on the diffusion of $K(t)$.

We consider a triatomic molecule with the total energy below the dissociation limit, total angular momentum $J$ and some initial value $K(0)>0$ close to the separatrix $K^{s x}$, as in Fig. 10. Since along the separatrix the value of $K^{s x}$ changes from 0 (at $\tau= \pm \infty)$ to $J\left(I_{1} / I_{2}\right)$ (at $\left.\tau=0\right)$, see Eq. (37), we use $K^{s x} \approx 0.5 J\left(I_{1} / I_{2}\right)$ in the equations below. At the initial time the distribution density of $K$ is a delta-function centered at

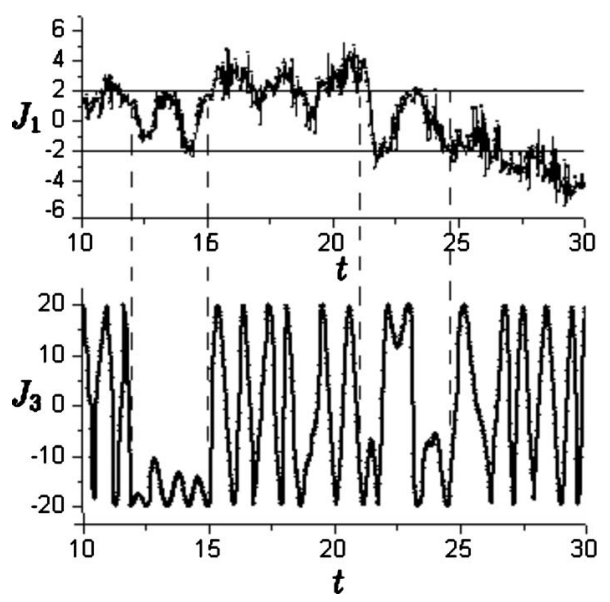

FIG. 9. Bifurcation of rotational behavior of ozone molecule near the separatrix (37) for $J=20$ and $E=-0.01 \mathrm{eV}$. The results of MD simulations are shown for $J_{1}$ and $J_{3}$ components of the total angular momentum $\mathbf{J}$, time is in picoseconds. Two horizontal lines of the $J_{1}(t)$ plot denote the additional zone of stable molecular rotations $\left|J_{1}\right|<\left(I_{1} / I_{2}\right) J$, in which $J_{1}$ can oscillate while $J_{3}$ is conserved, see text. Outside the zone, i.e., $\left|J_{1}\right|>\left(I_{1} / I_{2}\right) J, J_{1}$ is conserved while $J_{3}$ oscillates. Vibrational chaos by means of Coriolis coupling forces angular momentum $\mathbf{J}$ to jump between the two zones of stable rotations.

$K(0)$, as in Fig. 10(a). According to Eq. (36), the distribution density $\rho(K)$ at later times will spread out with the variance $\sigma^{2}=\left\langle(K-K(0))^{2}\right\rangle=2 D_{c}\left(J^{2}-K(0)^{2}\right) t$ and the mean value $\mu=\langle K\rangle=K(0)-(1+\sqrt{2}) K(0) D_{c} t$ changing linearly at initial times. At some time, the expanding distribution density $\rho(K)$ reaches the point $K^{s x}$, as in Fig. 10(b), which provides an opportunity for those trajectories $K(t)$ that appeared in the interval $\left(-K^{s x}, K^{s x}\right)$ to enter the region where $\mathbf{J}$ rotates around the $\xi_{3}$-axis and $K$ oscillates around the zero. Within a very short time [compared to the characteristic diffusion time $1 / D_{c}$ of $\left.K(t)\right], 2 \pi /\left(J / I_{3}\right)$, the interval $\left(-K^{s x}, K^{s x}\right)$ becomes uniformly populated due to the oscillations of $K(t)$ around zero and the distribution density should now have a form shown in Fig. 10(b). At later times the typical form of distribution density remains as the one shown in Fig. 10(c) with the $S_{1}$ area, defined in Fig. 10(d), "filled up" to the $y$-coordinate of spreading Gaussian $S_{0}$ at $K=K^{s x}$. The distribution density at initial times can be therefore represented by a Gaussian of variance $\sigma$ that is split at $K^{s x}$ in two parts, which are apart by a distance $2 K^{s x}$ and the interval $\left(-K^{s x}, K^{s x}\right)$ is filled uniformly and continuously. This simple explanation agrees with the results of MD simulations for ozone molecule shown in Figs. 10(e) and 10(f).

One can see from Fig. 10(d) that the asymmetry of the distribution function caused by the area $S_{1}$ may change the average values of $\langle K(t)\rangle$ and $\left\langle(K(t)-K(0))^{2}\right\rangle$, which otherwise would be equal to $\mu(t)$ and $\sigma(t)^{2}$, respectively. We know that at small values of $K$ the value of $\sigma$ changes with time as $O(J)$ while the value of $\mu$ changes as $O(K)$, i.e., the peak of the Gaussian moves much slower than it spreads. Therefore the dynamics of asymmetry of the distribution function due the presence of $S_{1}$ area is determined by $\sigma(t)$ alone. Since both $\sigma(t)$ and the asymmetry of distribution function change on the same time scale and $\sigma(t)$ increases with time, the effect of $S_{1}$-area on $\left\langle(K(t)-K(0))^{2}\right\rangle$ is negli- 

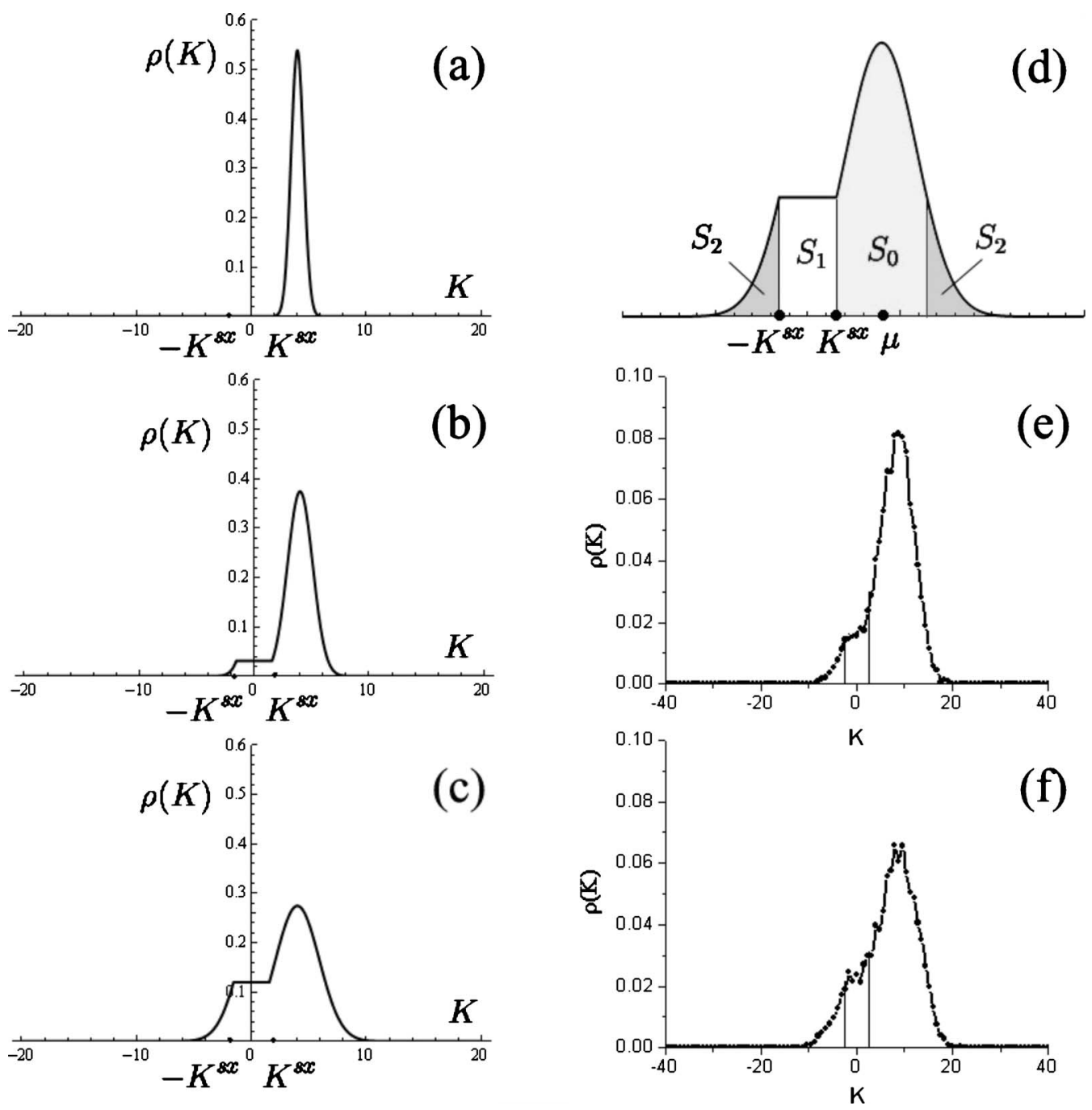

FIG. 10. Distribution densities $\rho(K)$ at initial times. Insets (a)-(c) schematically represent the change in distribution density as time goes from (a) to (c). Inset (d) denotes areas of $\rho(t)$ used in text. Insets (e) and (f) show the histogram of $\rho(t)$ at times 3.5 and 5.5 ps, respectively, obtained from MD simulation of ozone molecule with $J=40, K(0)=8$ and total energy $-0.01 \mathrm{eV}$. Vertical lines in (e) and (f) correspond to the interval $\left(-K^{s x}, K^{s x}\right)$.

gible: $\left\langle(K(t)-K(0))^{2}\right\rangle \approx\left(\sigma(t)+K^{s x}\right)^{2} \approx \sigma(t)^{2}$. Yet, the situation is different for the mean value $\langle K(t)\rangle$, the absolute value of which decreases with time as follows from the Appendix. In addition, $\langle K(t)\rangle$ changes much slowly, on the order of $O(K)$, than the change of the asymmetry of the distribution function, which is of the order of $O(J)$. Therefore the effect of the asymmetry of the distribution function due to the appearance of the $S_{1}$ area may have a significant effect on the dynamics of $\langle K(t)\rangle$. The latter may result in an effectively higher drift coefficient of $K$.

To estimate the increase in the drift coefficient we use simple geometrical considerations. If we use the notations $S_{0}$, $S_{1}$, and $S_{2}$ for the corresponding areas of the distribution density shown in Fig. 10(d), then the mean value of $K$ is

$$
\begin{aligned}
\langle K\rangle= & \mu\left(\frac{S_{0}}{S_{0}+S_{1}+S_{2}}\right)+0\left(\frac{S_{1}}{S_{0}+S_{1}+S_{2}}\right) \\
& +\left(\mu-K^{s x}\right)\left(\frac{S_{2}}{S_{0}+S_{1}+S_{2}}\right)
\end{aligned}
$$

where $\mu, 0$, and $\left(\mu-K^{s x}\right)$ are the corresponding mean values of $K$ for the areas $S_{0}, S_{1}$, and $S_{2}$, respectively. Rearranging Eq. (38) we obtain

$$
\langle K\rangle=\mu-\frac{\mu S_{1}+K^{s x} S_{2}}{S_{0}+S_{1}+S_{2}},
$$

i.e., $S_{1}$ and $K^{s x}$ shift $\langle K\rangle$ toward zero on the time scale of $\sigma(t)$, which is the reason for faster drift coefficient of $K(t)$. From simple geometrical considerations, as in Fig. 10(d), we find the normalized areas

$$
S_{1}=\frac{2 K^{s x} \exp \left[-\left(\mu-K^{s x}\right)^{2} / 2 \sigma^{2}\right]}{\sqrt{2 \pi} \sigma+2 K^{s x} \exp \left[-\left(\mu-K^{s x}\right)^{2} / 2 \sigma^{2}\right]},
$$

$$
S_{2}=\frac{\sqrt{2 \pi} \sigma\left(1-\operatorname{erf}\left[\left(\mu-K^{s x}\right) / \sqrt{2} \sigma\right]\right)}{\sqrt{2 \pi} \sigma+2 K^{s x} \exp \left[-\left(\mu-K^{s x}\right)^{2} / 2 \sigma^{2}\right]} .
$$

Given with $\quad \sigma=\sqrt{2 D_{c}\left(J^{2}-K(0)^{2}\right) t}$ and $\mu=K(0)$ $-(1+\sqrt{2}) K(0) D_{c} t$, we find the correction to the mean value of $K(t)$ obtained from a symmetric top model, 

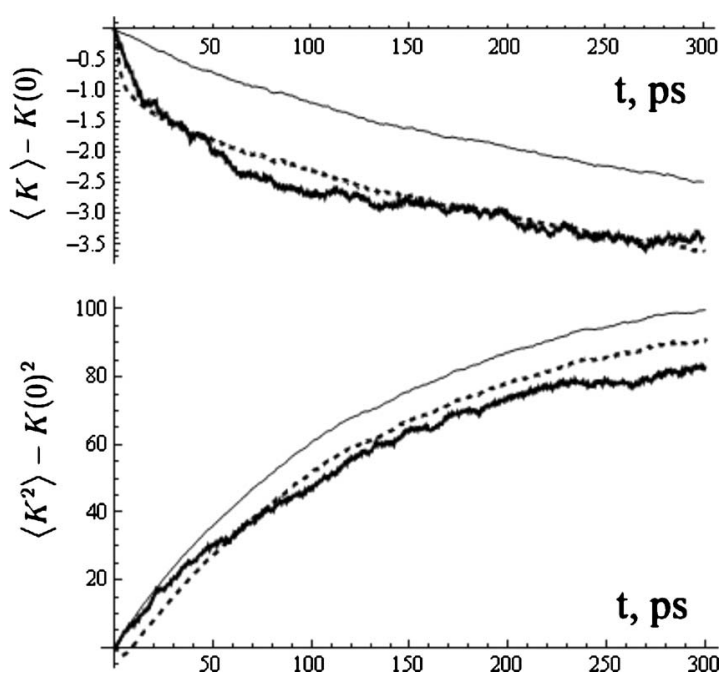

FIG. 11. The figure illustrates correction to the symmetric top approximation for ozone molecule with $J=20, K(0)=4$, and $E=-0.025 \mathrm{eV}$. Solid thick lines in both plots represent the results of MD simulations; solid thin lines are obtained from integration of stochastic Eq. (36); dashed lines are the results of stochastic Eq. (36) corrected by the value Eq. (41).

$$
\mu_{\text {corr }}(t)=-\mu S_{1}-K^{s x} S_{2}, \quad K(0)>0 .
$$

One needs to add this correction to the mean values of $\langle K(t)\rangle$ obtained from integration of stochastic Eq. (36). Equation (41) is approximate since the stochastic equation (36) is nonlinear and, in general, does not result in Gaussian distributions of $K$ at all times.

An acceleration of the drift coefficient of $K$ due to the presence of the correction term (41) influences the vibrational-rotational energy exchange as seen in the relation $(d / d t) E_{\text {rotation }} \sim(d / d t)\left\langle K(t)^{2}\right\rangle=(d / d t)\left\langle(K(t)-K(0))^{2}\right\rangle$ $+2 K(0)(d / d t)\langle K(t)\rangle=(d / d t) \sigma(t)^{2}+2 K(0)((d / d t) \mu(t)$ $\left.+(d / d t) \mu_{\text {corr }}(t)\right)$. At initial times and small values of $K(0)$ the derivative $(d / d t) \mu_{\text {corr }}(t)$ is significant, which means that for molecules with low values of $K(0)$ the rotational-vibrational energy exchange happens more slowly in the asymmetric top model than in the symmetric top approximation [since $(d / d t) \sigma(t)^{2}>0$ and $\left.K(0)(d / d t) \mu_{\text {corr }}(t)<0\right]$. Examples are given in Fig. 11. We note here that one should not confuse the symmetry of molecular ellipsoid of inertia with the isotopic symmetry of a triatomic molecule.

Nevertheless, the correction to the symmetric top approximation described in the present section is more an exception than the rule. Indeed, all the discussion above referred to the specific case of initial conditions in the form of delta-function centered at $K=K(0)$ and energies below the dissociation threshold. First, no real conditions will provide a distribution function of $K$ which is biased or asymmetric in $K(0)$, i.e., there is no physical reason to consider initial distribution functions of $K$ with $K(0) \neq 0$. Second, the case of total energy below the dissociation threshold is of low practical interest since highly vibrationally excited triatomic molecules formed in atom-diatom collisions have total energy above the dissociation threshold. Molecules with low $K(0)$ and total energy above dissociation have the highest possible vibrational energy and can easily dissociate. Therefore the distribution density $\rho(K)$ is expected to be mostly depleted on the interval $\left(-K^{s x}, K^{s x}\right)$ and therefore the effect of the asymmetry of molecular inertia ellipsoid will be minor.

\section{THE NON-RRKM EFFECTS}

We can now estimate the rate of diffusion of $K$ using Einstein's law of diffusion $\left\langle K^{2}\right\rangle=2 D t$. The typical angular momentum of an ozone molecule at room temperatures was estimated previously to be $J=17 \hbar$; it follows from Eqs. (31) and (43) that the diffusion constant $D \approx J^{2} D_{c}=0.3 \hbar^{2} \mathrm{ps}^{-1}$. For the typical experimental conditions where the massindependent effect is still observed, at 1 atm, the frequency of collisions is $5 \times 10^{9}$ collisions $/ \mathrm{s}^{3}$ which makes the average lifetime of ozone molecule of the order of 200 ps. During this time $K$ changes on average by $\sqrt{\left\langle K^{2}\right\rangle}$ $=\sqrt{0.3 \hbar^{2} \times 200}=7.7 \hbar$, which constitutes $45 \%$ of $J$. The present analysis thus indicates that for ozone molecule $K(t)$ is neither adiabatic (constant) nor active (rapidly varying). This result implies that the intramolecular dynamics of ozone molecule is essentially of a non-RRKM type. In particular, one of the major consequences of such a non-RRKM behavior is the possibility for $K(t)$ to diffuse into the region, which is not allowed in the usual adiabatic RRKM approximation and which is sometimes called a closed channels domain. ${ }^{17}$ In the adiabatic RRKM approximation ozone molecules with the total energy above the energy of dissociation yet with a total vibrational energy below the dissociation barrier $E_{\text {diss }}$ (closed states), $E_{\text {vib }}=E-E_{\text {rot }}(K)<E_{\text {diss }}$, or in other words with the values of $|K|$ greater than $K_{\max }$

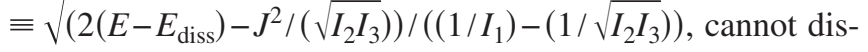
sociate without an additional exciting collision. In the same way, excited ozone molecules with $|K|<K_{\max }$ and $E_{\text {vib }}$ $>E_{\text {diss }}$ cannot be stabilized (enter closed states region $|K|$ $\left.>K_{\text {max }}\right)$ without an additional event of de-excitation. Yet, following from the present analysis, the molecule actually does not need any collision to enter the domain of closed states or to return back: $K(t)$ simply diffuses there obeying the diffusion law in Eq. (36), as in Fig. 5. The possibility of an ozone molecule dissociating thus is determined by the value of $K$. The values of $K$ obey the diffusion law in Eq. (36); therefore the possibility of a molecule dissociating is controlled by the diffusion process (36). We performed numerical tests that showed that vibrationally excited ozone molecules with a total energy above the dissociation threshold do not dissociate until the values of $|K|$ become less than $K_{\max }$, as shown in Fig. 5. We also observed reversible "random walk" of $K(t)$ to and from the closed states in numerical MD simulations with the representative numerical trajectory shown in Fig. 5; one can also see the distribution functions of $K$ in Fig. 12 and note that $K$ spans all possible values from $-J$ to $J$ at longer times. The mechanism of the $K$-diffusion controlled dissociation of ozone molecules discussed in the present section also agrees well with the direct numerical simulation for the statistics of lifetimes of vibrationally excited ozone molecules. ${ }^{18}$

\section{EFFECTS OF ISOTOPIC SYMMETRY}

In principle, the isotopic symmetry of the molecule can directly influence Coriolis noise $\delta J_{c}$. Since the strength of 

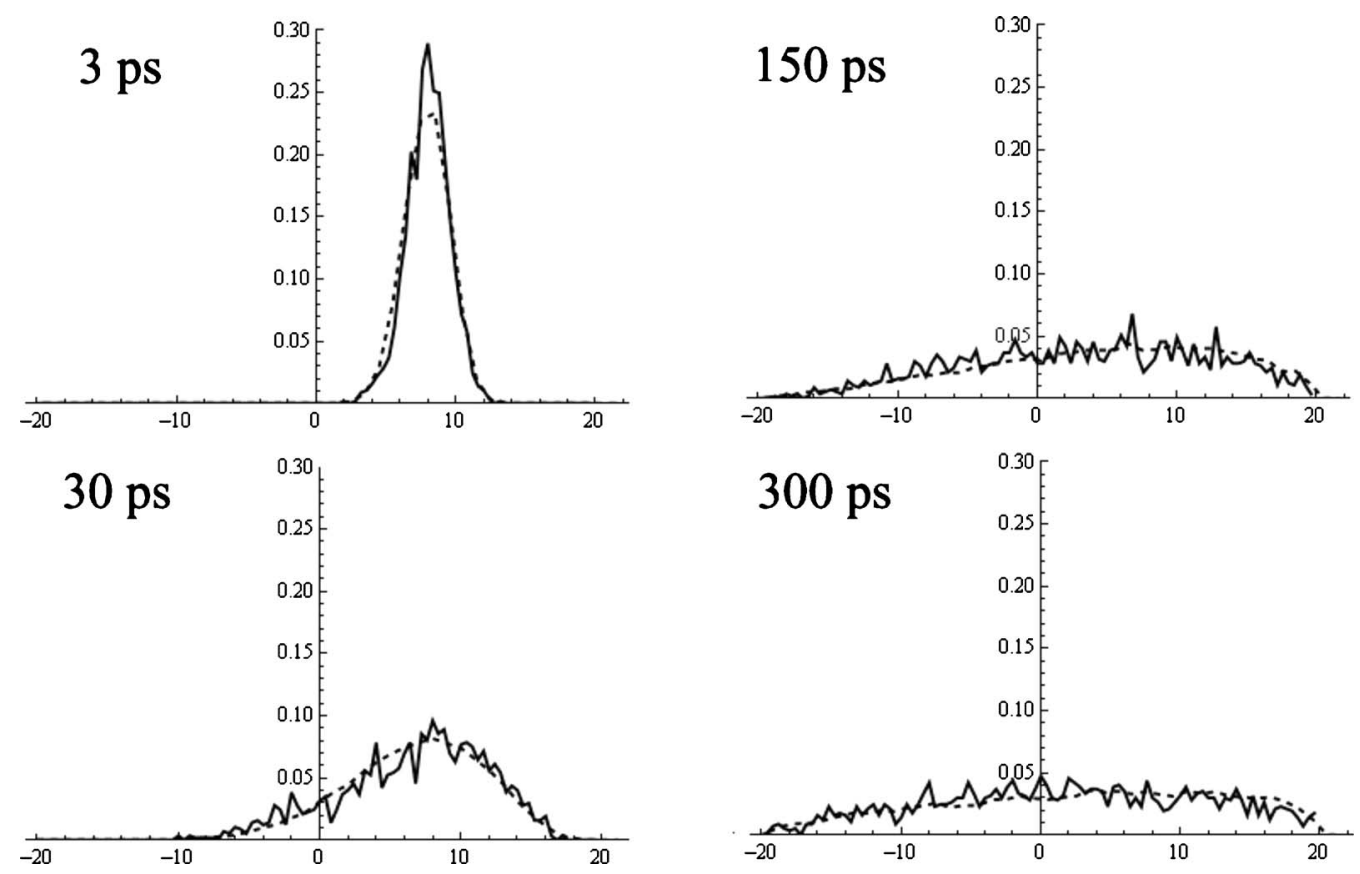

FIG. 12. Evolution of distribution density $\rho(K)$ for ozone molecule with $J=20, K(0)=8$, and total energy $E=-0.025 \mathrm{eV}$. Solid lines are the results of MD simulations, and dashed lines are the result of integration of stochastic Eq. (36).

Coriolis coupling should depend on the symmetry of ozone molecule, as in Fig. 3, the diffusion of $K$ is expected to depend on the symmetry of ozone molecule as well. In a following paper $^{18}$ we show that the diffusion of $K$-component of angular momentum defines the statistics of lifetimes of excited ozone molecules. However, no significant effect of symmetry on the diffusion of $K$ was observed, similar to the classical numerical results for different isotopomers of ozone shown in Ref. 8. We speculate that the symmetry effect of Coriolis coupling can appear in quantum mechanical analysis of the model which is a subject of ongoing study.

\section{NUMERICAL METHODS}

We used MD simulations to numerically compute correlation functions and to find the diffusion coefficient $D_{c}(E)$. We considered a model of ozone molecule $O^{16} O^{16} O^{16}$ with the interatomic potential ${ }^{19}$ used in Refs. 9 and 10. At each time step we calculated inertia tensor ${ }^{14}$

$I_{i k}=\left(\begin{array}{ccc}\sum m\left(y^{2}+z^{2}\right) & -\sum m x y & -\sum m x z \\ -\sum m y x & \sum m\left(x^{2}+z^{2}\right) & -\sum m y z \\ -\sum m z x & -\sum m z y & \sum m\left(x^{2}+y^{2}\right)\end{array}\right)$,

diagonalized it, and found its principal moments of inertia $I_{1}(t), I_{2}(t)$, and $I_{3}(t)$ and its principal axes $\xi_{1}, \xi_{2}$, and $\xi_{3}$. Projecting angular momentum $\mathbf{J}$ to the principal axes of inertia we kept track of components of angular momentum in body-fixed reference frame $F^{\prime}$. In particular, we were interested in the variance and the mean of $K(t)$. We performed microcanonical sampling of ozone molecules for given total energy $E$, angular momentum $J$, and a fixed initial value $K(0)$ of $K$-projection of angular momentum. We selected the values of $|K(0)|$ such that the diffusion of $K$ is primarily governed by $V(t)$-term in Eq. (20), i.e., we selected $K(0)$ far from its maximum value of $J$, where $U(t)$-term dominates over $V(t)$-term; we selected $|K(0)|$ far from $K^{s x}=\left(I_{1} / I_{2}\right) J$ to eliminate the effects of asymmetry of inertia ellipsoid. We propagated trajectories for about 5-10 ps and calculated the mean square deviation $\left\langle(K(t)-K(0))^{2}\right\rangle$ averaged over 1000 trajectories, yielding the results in Fig. 13. The short time for the trajectory propagation and the slowness of the diffusion rate of $K$ guaranteed that the average value of $K$ along the whole trajectory remained equal to $K(0)$. We then obtained

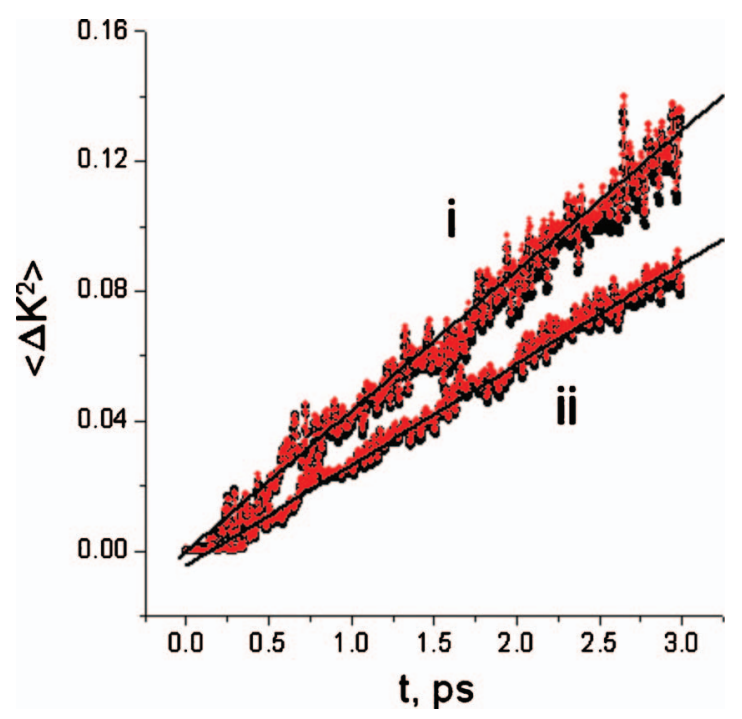

FIG. 13. Mean-square deviation of $K$ as a function of time. Results (i) correspond to the case $E=E_{\mathrm{diss}}+0.015 \mathrm{eV}, J=7.6 \hbar$, and $K(0)=4.5 \hbar$. Results (ii) correspond to the case $E=E_{\text {diss }}+0.015 \mathrm{eV}, J=7.6 \hbar$, and $K(0)=6.1 \hbar$. Colored points correspond to the direct MD computation of $K(t)$, and black points correspond to the numerical solution $K(t)=-\int \delta J_{c}\left(t^{\prime}\right) J_{2}\left(t^{\prime}\right) / I_{3}\left(t^{\prime}\right) d t^{\prime}$ of the approximate differential Eq. (13) along the same MD trajectory. Slope of best fit line provides a diffusion coefficient $2 D(K(0))$. 


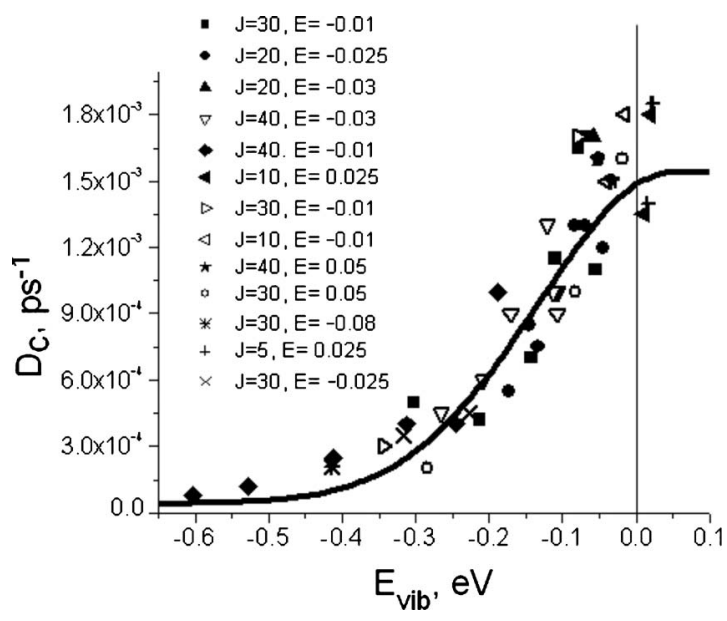

FIG. 14. Coefficient $D_{c}$ as a function of excess vibrational energy $E_{\mathrm{vib}}$ $-E_{\text {diss }}$ for ozone molecule. Vertical line represents zero excess energy $E_{\text {vib }}$ $-E_{\text {diss }}=0$. Each type of data point corresponds to particular value of $J$ and total energy $E$, in $\mathrm{eV}$, and different values of $K(0)$. The solid line represents the fitting curve (43).

diffusion coefficient $D(K(0))$ as $2 D=\lim _{t \rightarrow \infty}\left\langle K(t)^{2}\right\rangle / t{ }^{15}$ The coefficient $D_{c}$ was then calculated as $D_{c}=D(K(0)) /\left(J^{2}\right.$ $\left.-K(0)^{2}\right)$ and plotted in Fig. 14. The analytical function $D_{c}\left(E_{\mathrm{vib}}\right)$ that fits the numerical results shown in Fig. 14 is

$D_{c}(E)$

$$
=\left\{\begin{array}{l}
0.00004+0.0015 \exp \left(-15(E-0.05)^{2}\right), \quad E<0.05 \\
0.05, \quad E>0.05
\end{array}\right.
$$

with $E$ in units of $\mathrm{eV}$ and $D_{c}$ in units of $\mathrm{ps}^{-1}$. We used analytical expression (43) along with Eq. (32) to perform numerical simulation of stochastic Eq. (36) employing Heun's stochastic numerical scheme. ${ }^{20}$ The results of MD simulations and the corresponding theoretical results from stochastic integration of Eq. (36) are shown in Fig. 7. The plots of probability density from MD simulations and from the stochastic integration of Eq. (36) are compared in Fig. 12.

Figure 7(b) of the present paper is similar to the Fig. 2 in Ref. 8, yet our results are of higher curvature. As follows from the analysis in Appendix, nonlinear time behavior of $\langle K\rangle$ and $\left\langle K^{2}\right\rangle$ appears at higher values of $D_{c}$. We have run independent numerical simulations to compare the values of $D_{c}$ produced by the potential energy surface (PES) used in the present work and the potential surface used by Schinke group $^{8}$ obtained in a private communication. Our PES indeed yielded higher values of $D_{c}$ than the PES of Schinke et al. ${ }^{8}$

The moment of time when ozone molecule was considered as dissociated in Fig. 5 was taken as the moment of time when one of the valence bonds extended for more than $3 \mathrm{~A}$. It was shown in Refs. 9 and 10 that the transition state for ozone molecule corresponds to valence bond extensions on the order of $2.5 \mathrm{~A}$, thus at $3 \mathrm{~A}$ the ozone molecule can be considered dissociated.

\section{DISCUSSION}

In the present paper we have shown that for a triatomic molecule such as ozone the projection of rotational angular momentum on the principal axis of inertia $\xi_{1}$ shows diffusive behavior and derived an explicit stochastic equation that governs such diffusion. Vibrational chaos plays the role of perturbing force to the rotational dynamics of a nearsymmetric top molecule such as ozone. As a result, the $K$-component of the total angular momentum $\mathbf{J}$ of the top diffuses. This diffusion is shown in the present paper both analytically and numerically. Diffusion induced by chaos has been extensively studied in the past in the context of Arnold diffusion. ${ }^{21}$ In particular, the stochastic pump model ${ }^{21} \mathrm{em}$ ploys a similar idea of diffusion in nonlinear systems induced by coupling to stochastic regions of phase space, i.e., by coupling to the bath of chaotic motion. This model as well as its quantum version ${ }^{22,23}$ has been used previously to describe intramolecular energy flows in weakly chaotic molecules and has been also pointed as being relevant to the ozone problem in Refs. 7 and 24.

For the ozone molecule, the observed diffusion is fast enough to violate the assumption of adiabaticity of $K$ and slow enough to consider $K$ active. Thus one should expect non-RRKM effects in the process of ozone formation. The main reason for non-RRKM behavior resides in the fact that once an activated molecule is formed and its $|K|$ has diffused above $K_{\max }$, i.e., to the closed states domain, the ozone molecule cannot dissociate and waits until $K$ diffuses back to the domain of open states. During this time (diffusion controlled time) ozone molecule is likely to undergo a deactivating collision. Thus, the process of ozone formation depends on the Coriolis-driven diffusion of $K$. In a subsequent paper $^{18}$ we show that the observed diffusive energy exchange between the rotational and vibrational degrees of freedom in ozone molecule explains the nonstatistical distribution of lifetimes of the activated ozone molecules observed in Refs. 9 and 10.

\section{APPENDIX: APPROXIMATE ANALYTICAL SOLUTION OF EQ. (31)}

In this appendix we derive an approximate analytical time behavior of the first two moments of $K(t)$ in Eq. (31). The exact time behavior will be slightly modified by the $K$-dependence of the coefficient $D_{c}(K)$. Yet, for the present analysis we can approximate it with a constant $D_{c}$ $\approx D_{c}(K(0))$ from Eq. (43).

In the Stratanovich representation, the stochastic equation (31) is solved using regular rules of integration. ${ }^{15}$ Its solution is

$$
K(t)=J \sin \left(\sqrt{D_{c}} \int_{0}^{t} \xi\left(t^{\prime}\right) d t^{\prime}+C_{0}\right)
$$

where $C_{0}=\arcsin (K(0) / J)$. Representing the sine function as a difference of complex exponents and using the result for the average value of an exponential function ${ }^{15}$

$$
\left\langle\exp \left[k \int_{0}^{t} \xi\left(t^{\prime}\right) d t^{\prime}\right]\right\rangle=\exp \left[k^{2} t\right]
$$

we obtain 


$$
\langle K(t)\rangle=K(0) e^{-D_{c} t},
$$

which on the time scale less than $\tau \sim 1 / D_{c}$ can be described by the linear law

$$
\langle K(t)-K(0)\rangle=-K(0) D_{c} t .
$$

For the second moment of $K(t)$ using the same formula (A2), we have

$$
\left\langle K^{2}(t)\right\rangle=K(0)^{2}+\frac{1}{2}\left(J^{2}-2 K(0)^{2}\right)\left(1-e^{-4 D_{c} t}\right) .
$$

For times less than $\tau \sim 1 / 4 D_{c}$, expression (A5) results in the linear dependence

$$
\left\langle K^{2}(t)-K(0)^{2}\right\rangle=2 D_{c}\left(J^{2}-2 K(0)^{2}\right) t .
$$

Note that at $K(0)=J / \sqrt{2}$ the slope of $\left\langle K^{2}(t)-K(0)^{2}\right\rangle$ changes its sign.

\footnotetext{
${ }^{1}$ Y. Q. Gao and R. A. Marcus, Science 293, 259 (2001).

${ }^{2}$ B. C. Hathorn and R. A. Marcus, J. Chem. Phys. 111, 4087 (1999).

${ }^{3}$ B. C. Hathorn and R. A. Marcus, J. Chem. Phys. 113, 9497 (2000).

${ }^{4}$ K. Mauersberger, D. Krankowsky, C. Janssen, and R. Schinke, Adv. At., Mol., Opt. Phys. 50, 1 (2005).

${ }^{5}$ J. E. Heidenreich III and M. H. Thiemens, J. Chem. Phys. 78, 892 (1983).
}

${ }^{6}$ J. E. Heidenreich III and M. H. Thiemens, J. Chem. Phys. 84, 2129 (1986).

${ }^{7}$ R. Schinke, S. Y. Grebenshchikov, M. V. Ivanov, and P. Fleurat-Lessard, Annu. Rev. Phys. Chem. 57, 625 (2006).

${ }^{8}$ M. V. Ivanov, S. Y. Grebenshchikov, and R. Schinke, J. Chem. Phys. 120, 10015 (2004).

${ }^{9}$ N. Ghaderi, R. A. Marcus (to be submitted).

${ }^{10}$ N. Ghaderi, R. A. Marcus (to be submitted).

${ }^{11}$ D. M. Wardlaw and R. A. Marcus, Adv. Chem. Phys. 70, 231 (1988).

${ }^{12}$ R. A. Marcus, J. Chem. Phys. 20, 352 (1952); 20, 355 (1952).

${ }^{13}$ P. J. Robinson and K. A. Holbrook, Unimolecular Reactions (WileyInterscience, London, 1972).

${ }^{14}$ L. D. Landau and E. M. Lifschitz, Mechanics (Pergamon, Oxford, NY, 1989).

${ }^{15}$ H. Risken, The Fokker-Planck Equation: Methods of Solution (SpringerVerlag, Berlin, 1989).

${ }^{16}$ M. Kryvohuz and J. Cao, J. Phys. Chem. B, DOI:10.1021/jp102675y (2010).

${ }^{17}$ Y. Q. Gao and R. A. Marcus, J. Chem. Phys. 116, 137 (2002).

${ }^{18}$ M. Kryvohuz and R. A. Marcus, J. Chem. Phys. 132, 224305 (2010).

${ }^{19}$ A. J. C. Varandas and J. N. Murrell, Chem. Phys. Lett. 88, 1 (1982).

${ }^{20}$ J. L. García-Palacios and F. J. Lázaro, Phys. Rev. B 58, 14937 (1998).

${ }^{21}$ A. J. Lichtenberg and M. A. Lieberman, Regular and Stochastic Motion (Springer, New York, 1983).

${ }^{22}$ D. M. Leitner and P. G. Wolynes, Chem. Phys. Lett. 276, 289 (1997).

${ }^{23}$ D. M. Leitner and P. G. Wolynes, Phys. Rev. Lett. 79, 55 (1997).

${ }^{24}$ S. F. Deppe, U. Wachsmuth, B. Abel, M. Bittererova, S. Yu. Grebenshchikov, R. Siebert, and R. Schinke, J. Chem. Phys. 121, 5191 (2004). 